\title{
SMARTS OPTICAL AND INFRARED MONITORING OF 12 GAMMA-RAY BRIGHT BLAZARS
}

\author{
Erin Bonning ${ }^{1}$, C. Megan Urry ${ }^{1}$, Charles Bailyn ${ }^{2}$, Michelle Buxton ${ }^{2}$, Ritaban Chatterjee $^{2}$, Paolo Coppi $^{2}$, \\ Giovanni FossaTi ${ }^{3}$, Jedidah ISLER ${ }^{1}$, AND LaURa MARASCHI ${ }^{4}$ \\ ${ }^{1}$ Department of Physics and Yale Center for Astronomy and Astrophysics, Yale University, P.O. Box 208121, New Haven, \\ CT 06520-8120, USA; erin.bonning@yale.edu \\ ${ }^{2}$ Department of Astronomy and Yale Center for Astronomy and Astrophysics, Yale University, P.O. Box 208101, New Haven, CT 06520-8101, USA \\ ${ }^{3}$ Department of Physics and Astronomy, Rice University, Houston, TX 77005, USA \\ ${ }^{4}$ INAF - Osservatorio Astronomico di Brera, V. Brera 28, I-20100 Milano, Italy \\ Received 2011 August 19; accepted 2012 June 5; published 2012 August 9
}

\begin{abstract}
We present multiwavelength data for 12 blazars observed from 2008 to 2010 as part of an ongoing optical-infrared photometric monitoring project. Sources were selected to be bright, southern $\left(\delta<20^{\circ}\right)$ blazars observed by the Fermi Gamma-Ray Space Telescope. Light curves are presented for the 12 blazars in BVRJK at near-daily cadence. We find that optical and infrared fluxes are well correlated in all sources. Gamma-ray bright flat spectrum radio quasars (FSRQs) in our sample have optical/infrared emission correlated with gamma-rays consistent with inverse Compton-scattering models. In FSRQs, variability amplitude increases toward IR wavelengths, consistent with the presence of a thermal accretion disk varying on significantly longer timescales than the jet. In BL Lac objects, variability is mainly constant, or increases toward shorter wavelength. FSRQs have redder optical-infrared colors when they are brighter, while BL Lac objects show no such trend. Several objects show complicated color-magnitude behavior: AO $0235+164$ appears in two different states depending on its gamma-ray intensity. OJ 287 and 3C 279 show some hysteresis tracks in their color-magnitude diagrams. Individual flares may be achromatic or otherwise depart from the trend, suggesting different jet components becoming important at different times. We present a time-dependent spectral energy distribution of the bright FSRQ 3C 454.3 during its 2009 December flare, which is well fit by an external Compton model in the bright state, although day-to-day changes pose challenges to a simple one-zone model. All data from the SMARTS monitoring program are publicly available on our Web site.
\end{abstract}

Key words: black hole physics - BL Lacertae objects: general - galaxies: active - galaxies: jets - quasars: general

Online-only material: color figures, extended figures

\section{INTRODUCTION}

Blazars form a subclass of active galactic nuclei (AGNs) with a bright, relativistic jet viewed closely along our line of sight (Urry \& Padovani 1995). Blazars are often very luminous and violently variable over a large range of wave bands from radio to gamma-rays. Spectral energy distributions (SEDs) of blazars are characterized by two broad components: one peaking anywhere from infrared to X-ray frequencies, and a second peak at higher energies, from hard $\mathrm{X}$-rays to $\mathrm{TeV}$ gamma-rays. The radio to optical/UV emission in blazars is interpreted as synchrotron radiation by the energetic electrons in the jet (Konigl 1981; Urry \& Mushotzky 1982), while the mechanism of the high energy emission (i.e., $\mathrm{X}$-rays and gamma-rays) is less certain. It may be due to the inverse-Compton scattering of seed photons by the same relativistic electrons responsible for the synchrotron radiation (the so-called leptonic models; e.g., Böttcher 2007) or due to synchrotron radiation of protons co-accelerated with the electrons in the jet, interactions of these highly relativistic protons with external radiation fields, or proton-induced particle cascades (hadronic models; e.g., Mücke \& Protheroe 2001; Mücke et al. 2003).

Both leptonic and hadronic models can successfully explain the SEDs observed so far, but they have very different implications for the kinetic power of the jet and hence how it is produced and its influence on its environment. In the case of leptonic models, the low-energy seed photons may be the synchrotron photons produced within the jet (synchrotron self-Compton, SSC; Jones et al. 1974) or thermal emission from outside the jet. External Compton (EC) scenarios produce high energy emis- sion from upscattering photons from the accretion disk, broadline region (BLR), or dusty torus (Sikora et al. 1994; Dermer \& Schlickeiser 1993; Ghisellini \& Madau 1996; Tavecchio et al. 2000; Ghisellini \& Celotti 2001). The jet plasma may consist of electrons and protons, electrons and positrons, or a combination of the two (Ghisellini \& Tavecchio 2010; Sikora \& Madejski 2000).

Knowing the composition of the jet is necessary to deduce its kinetic power, which in turn reflects how it is launched, accelerated, and collimated. Studying the SED and its variation with time allows us to determine the radiation mechanism, and thus the physical parameters of the emission region, such as the magnetic field, particle number density, and bulk velocity of the plasma.

Until recently, blazar SED studies occurred primarily when the brightness of a blazar significantly increased in one or multiple wave bands. Due to the difficulty of coordinating large multiwavelength campaigns, data in other wavebands were often non-simultaneous. The near-continuous monitoring activity of the Large Area Telescope (LAT) instrument on board the Fermi Gamma-Ray Space Telescope, launched in 2008, provides the opportunity to study the variable SEDs of a large sample of blazars with truly simultaneous multi-frequency data. Although many blazars radiate most of their energy in the gamma-ray band, it is the characterization of both broad components of the blazar SEDs and their relative variation with time that allows us to infer the physics of these sources. Specifically, correlations between variations in gamma-ray flux and those at lower energies are useful indicators of the relative locations of emission regions and the radiation mechanism(s). 
Fermi/LAT provides regular and well sampled gamma-ray light curves of a large sample of blazars. Obtaining the same quality data at optical and infrared wavelengths is equally important. We use the meter-class telescopes of the Small and Moderate Aperture Research Telescope System (SMARTS) to carry out photometric monitoring of bright southern gamma-ray blazars on a regular cadence, at both optical and near-infrared wavelengths.

In this paper, we report on the Yale/SMARTS blazar monitoring program for the years 2008-2010. We present light curves for the blazars with the greatest coverage, and refer the reader to our Web site ${ }^{5}$ for the full data set. In addition, we report on gamma-ray/optical-IR cross-correlation functions for several blazars and show an example of how a variable SED has the potential to constrain the physics of a jet and/or accretion disk. Finally, we discuss the color-magnitude diagrams for blazars and how these can yield broad inferences about particle acceleration and radiative losses in blazar jets.

In Section 2, we present the sample selection and data reduction and present the multiwavelength light curves. In Section 3, we discuss the variability characteristics of the blazars in our sample, including cross-correlations between gamma-ray and optical-IR variations, frequency-dependent variability amplitude, and color-magnitude relations for our sample of blazars. We discuss SED fits for a sample blazar flare in Section 5. In Section 6, we present the summary and conclusions.

\section{SAMPLE SELECTION AND DATA ANALYSIS}

The SMARTS blazar sample was initially (in 2008) defined to include all LAT-monitored blazars on the initial public release list with declination $<20^{\circ}$. Prior to the launch of Fermi, the list of sources that were to have fluxes made publicly available consisted mainly of bright blazars observed by EGRET. Additional sources were added to our monitoring campaign as they were added to the public LAT source list or were the target of a multiwavelength campaign. We observed the 12 sources presented here with a cadence of approximately once every three days. Brighter or flaring sources were observed nightly. The SMARTS source list, including positions, redshifts, and observation time frame, is given in Table 1. Each of the blazars is identified by its spectral class: flat-spectrum radio quasar (FSRQ), low frequency-peaked BL Lac object (LBL), or high frequency-peaked BL Lac object (HBL). These form a rough sequence of decreasing continuum luminosity and emission line luminosity (Fossati et al. 1998). The most luminous blazars (FSRQ and LBL) have their synchrotron peak at IR/optical wavelengths and the inverse Compton peak in $\mathrm{GeV}$ gamma-rays so they form the bulk of our monitored sample.

The 12 blazars were observed with the SMARTS $1.3 \mathrm{~m}$ telescope and ANDICAM instrument (DePoy et al. 2003). ANDICAM is a dual-channel imager with a dichroic that feeds an optical CCD and an IR imager, which can obtain simultaneous data from 0.4 to $2.2 \mu \mathrm{m}$. Observations were taken in $B, V, R, J$, and $K$ bands, except for two sources, PKS $0528+134$ and $3 C 273$, for which $K$-band images were not obtained. The former was below the $K$-band detection limit; the latter, being very bright, had very short exposure times in optical bands. Since IR images are taken simultaneously with optical, there was only sufficient time for $J$-band images to be obtained for 3C 273. Additionally, spectra were obtained for a number of the brighter sources, using the SMARTS RCSPEC+1.5-meter

\footnotetext{
5 http://www.astro.yale.edu/smarts/fermi
}

Table 1

The SMARTS Blazar Sample

\begin{tabular}{lclcc}
\hline \hline Name & $\begin{array}{c}\text { R.A. and Decl. (J2000) } \\
\text { (h:m:s) (d:m:s) }\end{array}$ & Class & $z$ & $\begin{array}{c}\text { Dates } \\
\text { (MJD) }\end{array}$ \\
\hline PKS 0208-512 & $02: 10: 46.2-51: 01: 01$ & FSRQ & 1.003 & $54640-55282$ \\
AO 0235+16 & $02: 38: 38.9+16: 36: 59$ & LBL & 0.940 & $54662-55487$ \\
PKS 0528+134 & $05: 30: 56.4+13: 31: 55$ & FSRQ & 2.060 & $54701-55511$ \\
OJ 287 & $08: 54: 48.8+20: 06: 31$ & LBL & 0.306 & $54777-55500$ \\
3C 273 & $12: 29: 06.7+02: 03: 09$ & FSRQ & 0.158 & $54603-55412$ \\
3C 279 & $12: 56: 11.1-05: 47: 21$ & FSRQ & 0.536 & $54603-55416$ \\
PKS 1406-076 & $14: 08: 56.5-07: 52: 27$ & FSRQ & 1.494 & $54501-55385$ \\
PKS 1510-08 & $15: 12: 50.5-09: 06: 00$ & FSRQ & 0.360 & $54603-55464$ \\
PKS 1622-29 & $16: 26: 06.0-29: 51: 27$ & FSRQ & 0.815 & $54501-55481$ \\
PKS 1730-130 & $17: 33: 02.6-13: 04: 49$ & FSRQ & 0.902 & $54603-55494$ \\
PKS 2155-304 & $21: 58: 52.0-30: 13: 32$ & HBL & 0.112 & $54603-55557$ \\
3C 454.3 & $22: 53: 57.7+16: 08: 54$ & FSRQ & 0.859 & $54640-55545$ \\
\hline
\end{tabular}

Notes. Coordinates and redshifts from NED (http://ned.ipac.caltech.edu/; the NASA/IPAC Extragalactic Database (NED) is operated by the Jet Propulsion Laboratory, California Institute of Technology, under contract with the National Aeronautics and Space Administration). Dates indicate limits of $R$-band observations reported here. Other bands may have slightly different date ranges.

telescope. These data will be discussed in forthcoming paper (J. Isler et al. 2012, in preparation).

Optical data were bias-subtracted, overscan-subtracted, and flat fielded using the CCDPROC task in IRAF. Infrared data were sky-subtracted, flat fielded, and dithered images combined using in-house IRAF scripts. Slight blemishes reflecting the dither pattern are apparent in the final images, but introduce at most a $0.1 \%$ photometric error.

Optical and infrared aperture photometry was performed using the PHOT task in IRAF. Non-variable comparison stars with comparable magnitude to the blazar were chosen in each field. The raw photometry of comparison stars in the field of the blazar was calibrated using photometric zero points that were measured from ANDICAM observations during 2008-2009 of optical (Landolt 1992) and near-infrared (Persson et al. 1998) primary standards for each filter, correcting for atmospheric extinction derived from all the standards taken together. The number of photometric nights available for the calibration for each field and each filter differs, but ranges from 40 to 128 nights in the optical and 13 to 98 nights for the near-infrared. The average of the comparison stars was used as a basis of differential photometry with respect to the blazar for all observations.

We compared our calibrated comparison star magnitudes to values reported in the literature where available. In the large majority of cases, our values agree with previous photometric sequences measured for these fields to within a typical $1 \sigma$ error of $\sim 0.05$ mag with an occasional discrepancy of $\sim 0.1$ mag. Our optical magnitudes for the comparison stars in the field of AO 0235+164 are consistent with values reported by Smith et al. (1985), Fiorucci et al. (1998), and González-Pérez et al. (2001). In the field of PKS 0528+134, our comparison star magnitudes match extremely well with those published by González-Pérez et al. (2001) with the exception of the $V$ band for star 1 , where we differ by $0.1 \mathrm{mag}$. When compared to the values published by Raiteri et al. (1998), our result agree in the $R$ band; however our $B$ and $V$ magnitudes are fainter by $0.1 \mathrm{mag}$ in $B$ and 0.2 mag in $V$ compared with those of Raiteri et al. In the case of OJ 287, our comparison stars are in good agreement with González-Pérez et al., with the largest discrepancy being $\sim 0.07$ mag. Likewise, our $V$ and $R$ measurements for the comparison stars for OJ 287 are in close agreement with those measured by Fiorucci \& Tosti 
(1996, who do not report the $B$ band.) The comparison star of 3C 273 is within uncertainties reported by Smith et al. (1985). 3C 279 is in close agreement with the values published by Smith $\&$ Balonek (1998) with the $R$ band being the only one to differ by more than $3 \sigma$ (0.1 mag). Our comparison stars for PKS 1510089 generally agreed within the uncertainties with the values published by González-Pérez et al. (2001), with the exception of the $B$ band for our star 1 , which differed by 0.1 mag. In the field of PKS 1622-297, our stars 1 and 2 are stars 10 and 14 of González-Pérez et al. (2001), and they are in excellent agreement in the $V$ and $R$ bands. (González-Pérez et al. do not report the $B$ band for this source). The comparison star in our field for PKS 2155-304 is in excellent agreement with the photometry of Hamuy \& Maza (1989). In the case of 3C 454.3, comparison star magnitudes have been reported for this wellstudied source also by Angione (1971) and Fiorucci et al. (1998). Our results agree extremely well with the results of Fiorucci et al. (1998) in $V$ and $R$, and again find a $\sim 0.1$ mag (fainter) discrepancy with Raiteri et al. (1998) in $B$, a discrepancy that only occurs for some stars reported by Angione (1971).

In general, we find that in those sources with multiple photometric sequences available (PKS1510-089, 3C 273, 3C 454.3), published comparison star values frequently differ on the level of $\sim 0.1$ magnitude. This is consistent with a comparison of our data to the literature. For our remaining sources, PKS 0208-512, PKS 1406-076, and PKS 1730-130, existing photometric values for comparison stars (given, for example, by the finding charts at http://www.lsw.uni-heidelberg.de/projects/extragalactic/charts) are taken from the USNO B1.0 catalog (Monet et al. 2003). Our calibrated magnitudes are generally fainter than USNO by 0.2-0.4 mag. The USNO B1.0 catalog was designed to be a proper-motion catalog. Although photometry was obtained, it has an accuracy of 0.3 mag (Monet et al. 2003), which is significantly greater than the errors found for our photometry.

The optical and IR light curves for the monitored blazars through 2010 July are shown in Figure 1. (At the time of writing, some third-year SMARTS data are already available online but the basic scientific results do not change for the 12 blazars discussed here.) These figures include daily and weekly gamma-ray fluxes from the Fermi/LAT public light curves for those sources bright enough in the gamma-ray that these are measurable. ${ }^{6}$ The optical and infrared light curves and calibrated magnitudes are available online at the Yale SMARTS Blazar site ${ }^{5}$. Optical and infrared finding charts are shown in Appendices A and B. Calibrated magnitudes for the comparison stars in each blazar field are given in Appendix C.

The error in calibrating the secondary star magnitudes was found by calculating the $1 \sigma$ standard error of the mean over the number of photometric nights mentioned above. Results that were greater than $\pm 3 \sigma$ from the mean were rejected and the mean and $\sigma$ were recalculated. This procedure was repeated until no more rejections were made. The resulting errors are given in Tables 3 and 4. These errors do not account for systematic errors associated with effects such as the difference in effective filter responses between SMARTS and the standard system. Such systematics are likely to contribute a few hundredths of a magnitude of calibration error.

Previous work with SMARTS photometry (Buxton et al. 2012) has led to an understanding of the errors in differential photometry for point sources as a function of count rate.

\footnotetext{
6 List of Fermi/LAT monitored sources is at http://fermi.gsfc.nasa.gov/ssc/data/access/lat/msl_lc/.
}

Based on this work, we find errors as low as $0.01 \mathrm{mag}$ in the optical and $0.02 \mathrm{mag}$ in the IR for bright sources $(<16 \mathrm{mag}$ in the optical and $<13 \mathrm{mag}$ in IR), and up to ten times that for sources near the detection limit, which varies depending on the exposure time. We note that these errors are random errors in the individual points, rather than systematic offsets in the magnitude system, as is the case for the calibration errors.

\section{RESULTS AND DISCUSSION}

\subsection{Multiwavelength Cross-correlation Results}

Every blazar in our sample is highly variable in gamma-rays and, in many cases, in the optical/IR as well; only 3C273 shows minimal variations in the optical/IR. The optical, near-infrared and gamma-ray light curves are shown in Figure 1.

We investigate the correlation between optical and near-IR bands through the discrete correlation function (DCF; Edelson \& Krolik 1988) with the corrections of White \& Peterson (1994). Figure 2 shows the $B-J$ band DCF for each source in our sample. (We also show the $R-J$ band DCF for PKS 0528+134. This is the only source for which the DCF was significantly different in shape between bands.) Examining the DCFs for each source we find that, apart from 3C 273, which has no strong variations, all DCFs have a peak at zero lag. No source shows a significant peak at any non-zero lag. Note that given our daily cadence, we are sensitive only to lags longer than a couple of days. In general, this correlation between optical and near-IR bands has been seen historically, e.g., in AO 0235+164 (Raiteri et al. 2006), in PKS 2155-304 (Paltani et al. 1997, who do measure a $\sim 40$ minute lag in longer wavelengths), and in 3C 279 (Hartman et al. 1996; Wehrle et al. 1998). Correlation is also seen between optical and UV wavelengths in the LBL OJ 287 (Pian et al. 1995).

Figure 3 shows two examples of the correlation between optical ( $B$ band) and IR ( $J$ band) for two blazars: PKS 2155-304 (an HBL, Figure 3(a)), and 3C 279 (an FSRQ, Figure 3(b)). Figure 3(b) also shows distinct tracks that reflect changes in the optical/IR spectral shape of 3C 279 over time (cf. Figure 5(b)).

Six blazars-four FSRQs, one LBL, and one HBL-were bright enough during 2008-2010 to be regularly detected by Fermi LAT in one-day time bins. The gamma-ray-infrared discrete correlation functions for the these sources are shown in Figure 4). In three cases, the optical-IR variability has been shown to be reasonably well-correlated with the gamma rays: 3C454.3 (Bonning et al. 2009), PKS 1510-089 (Marscher et al. 2010), and AO 0235+164 (Agudo et al. 2011). The FSRQs 3C 273 and 3C 279 show weak (if any) correlations between infrared and gamma-rays, while the optical/IR emission of the HBL PKS 2155-304 is clearly uncorrelated with gamma-ray emission. The strongly disk-dominated FSRQ 3C 273, shows little to no variability in the optical/IR bands (fluctuations on the order of $1 \%$ ) compared to the variations by a factor of several to 10 in gamma-rays. The small fractional variation at optical/ IR frequencies in 3C 273 may be due to the relatively large luminosity of the accretion disk (Ramos et al. 1997), since the disk probably does not vary in the optical/IR over timescales as short as the gamma rays. For 3C 279, optical/IR-gamma ray correlations have been reported by Abdo et al. (2010), who note the coincidence of a gamma-ray flare with a strong change in optical polarization angle. However, our SMARTS data show larger amplitude changes (by several orders of magnitude) than the gamma-ray data, over both the first and second years of Fermi 

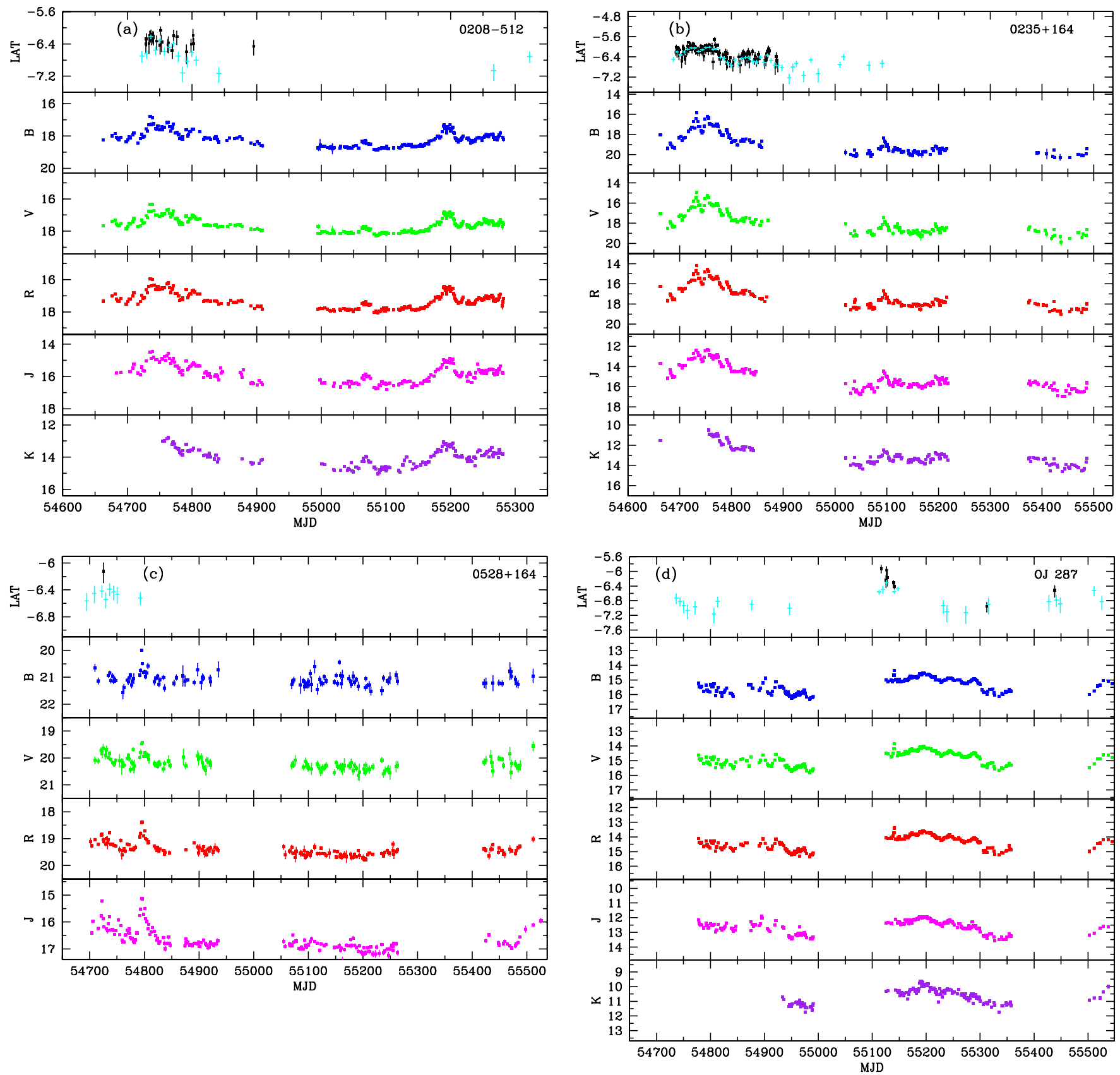

Figure 1. SMARTS optical and near-infrared light curves for (a) PKS 0208-512, (b) AO 0235+164, (c) PKS 0528+134, (d) OJ 287, (e) 3C 273, (f) 3C 279, (g) PKS 1406-076, (h) PKS 1510-089, (i) PKS 1622-297, (j) PKS 1730-130, (k) PKS 2155-304, and (1) 3C 454.3. Fermi/LAT fluxes are taken from the public daily (black points) and weekly (cyan points) light curves (except panel (g), taken from the public weekly (cyan points) light curves only) and are in units of log (photons $\mathrm{s}^{-1} \mathrm{~cm}^{-2}$ ).

(A color version of this figure is available in the online journal.)

observations. The one HBL with high enough gamma-ray flux to appear consistently in the LAT daily fluxes, PKS 2155-304, shows variations of a factor of 2-3 in gamma-rays, along with longer-timescale variations in the optical-IR, but the two appear to be uncorrelated.

The close correlation of optical/IR and gamma-ray fluxes strongly favors leptonic models over hadronic models. In the latter, the two broad components of the SED should vary almost independently, while in leptonic models, the two peaks are should vary together. In the FSRQs, the peak of the low energy component of the SED is in the far-IR, longward of the SMARTS bands, while the high-energy component peaks in or near the Fermi LAT energy range. In terms of simple homogeneous leptonic models, the SMARTS data generally sample emission from higher energy electrons than the Fermi LAT data. (this can obviously change if the SED peak moves, as in a major acceleration event, e.g., Pian et al. 1998, for the case of Mrk 501) More realistic electron distributions and/or geometries will complicate this picture, but in any case, as discussed in Section 4 , there are multiple plausible reasons for variations in the strength of the optical-IR versus gamma-ray correlations among the FSRQs in our sample.

\subsection{Excess Variance}

Most blazars are strongly variable at gamma-ray energies and in the optical/IR. To quantify and compare the degree of variability in different blazars, and in different bands for the 

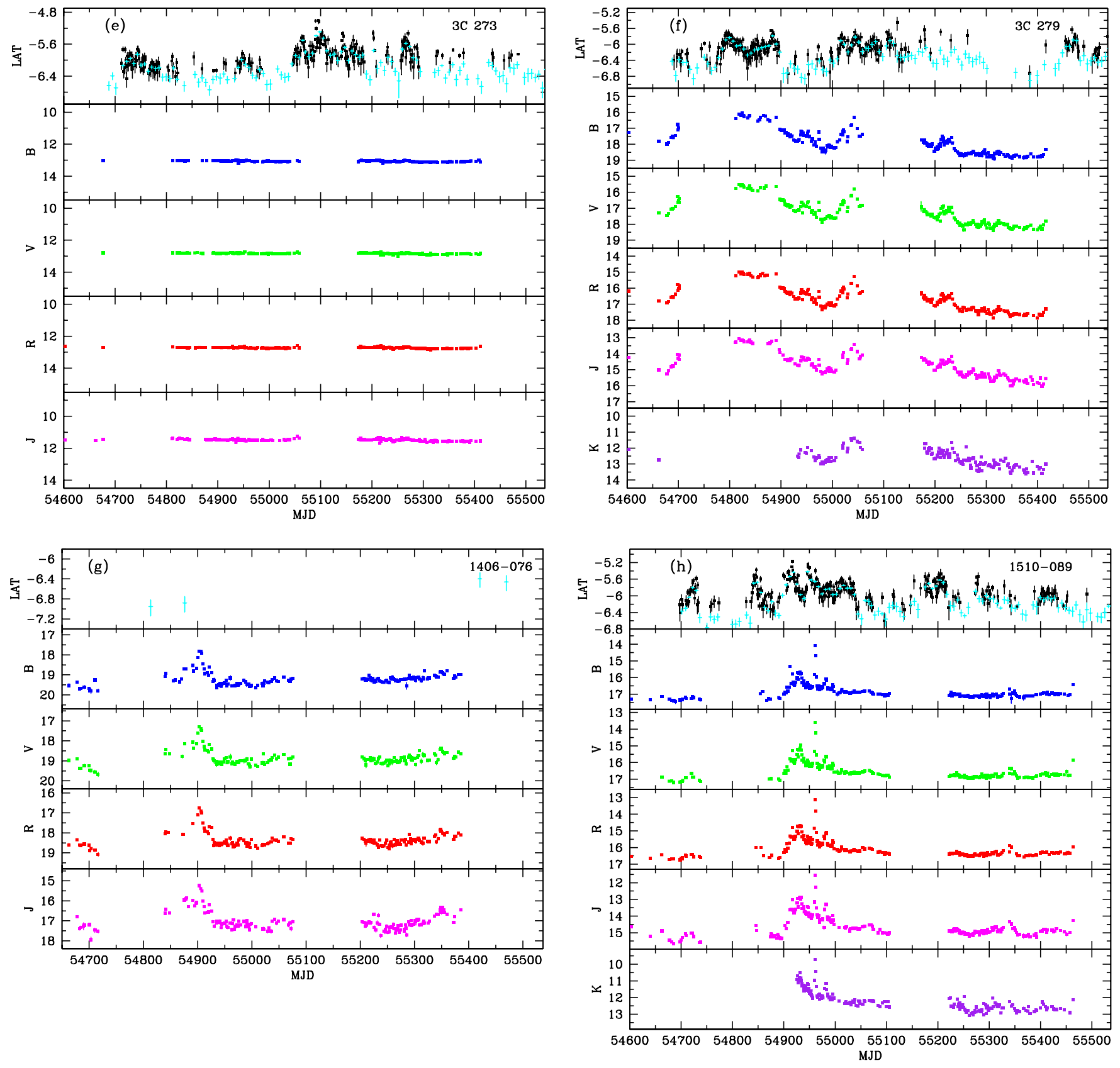

Figure 1. (Continued)

same blazar, we calculate the "excess variance," which is the fractional root-mean-squared variability amplitude normalized by the mean flux:

$$
F_{\mathrm{var}}=\sqrt{\frac{\mathrm{S}^{2}-\overline{\sigma_{\mathrm{err}}^{2}}}{\bar{x}^{2}}},
$$

where $\mathrm{S}$ is the variance, $\sigma_{\mathrm{err}}$ is the observational uncertainty, and $\bar{x}$ is the mean of the data (Nandra et al. 1997; Edelson et al. 2002; Vaughan et al. 2003). The values of $F_{\text {var }}$ calculated for SMARTS and Fermi LAT data are listed in Table 2. In cases where $K$-band observations did not cover the same time periods as the other filters, we omit the $K$-band values.

The IR/optical/UV emission from blazars is a combination of thermal emission from a (possibly weak) accretion disk and relativistically beamed nonthermal emission from a jet. The disk emission peaks in the UV band so its relative contribution in optical/IR bands decreases toward longer wavelengths. Since the disk emission comes from $\gtrsim 10^{4} r_{g}$ (Malkan 1983; Sun \& Malkan 1989), it is almost certainly less rapidly variable than from the jet. Therefore, on the timescales considered here, we expect the variability amplitude for FSRQs (blazars with strong emission lines implying a radiatively efficient accretion disk) in the optical/IR to increase with wavelength, i.e., infrared bands to vary more than the optical. In general, this effect is seen in Table 2. For the FSRQs, $J$-band is more variable than $B$-band in all objects except 3C 279, in which the trend is reversed (also reported by Wehrle et al. 1998). In Section 4 and Figure 5(f), it is shown that the changes in spectral shape of 3C 279 are also not consistent with a simply varying jet component overlaid on a constant blue accretion disk. The BL Lac objects do not show greater variability toward the IR: The two LBLs are more variable toward the $B$-band, and the HBL PKS 2155-304 shows no frequency-dependent variability changes. BL Lac objects, 

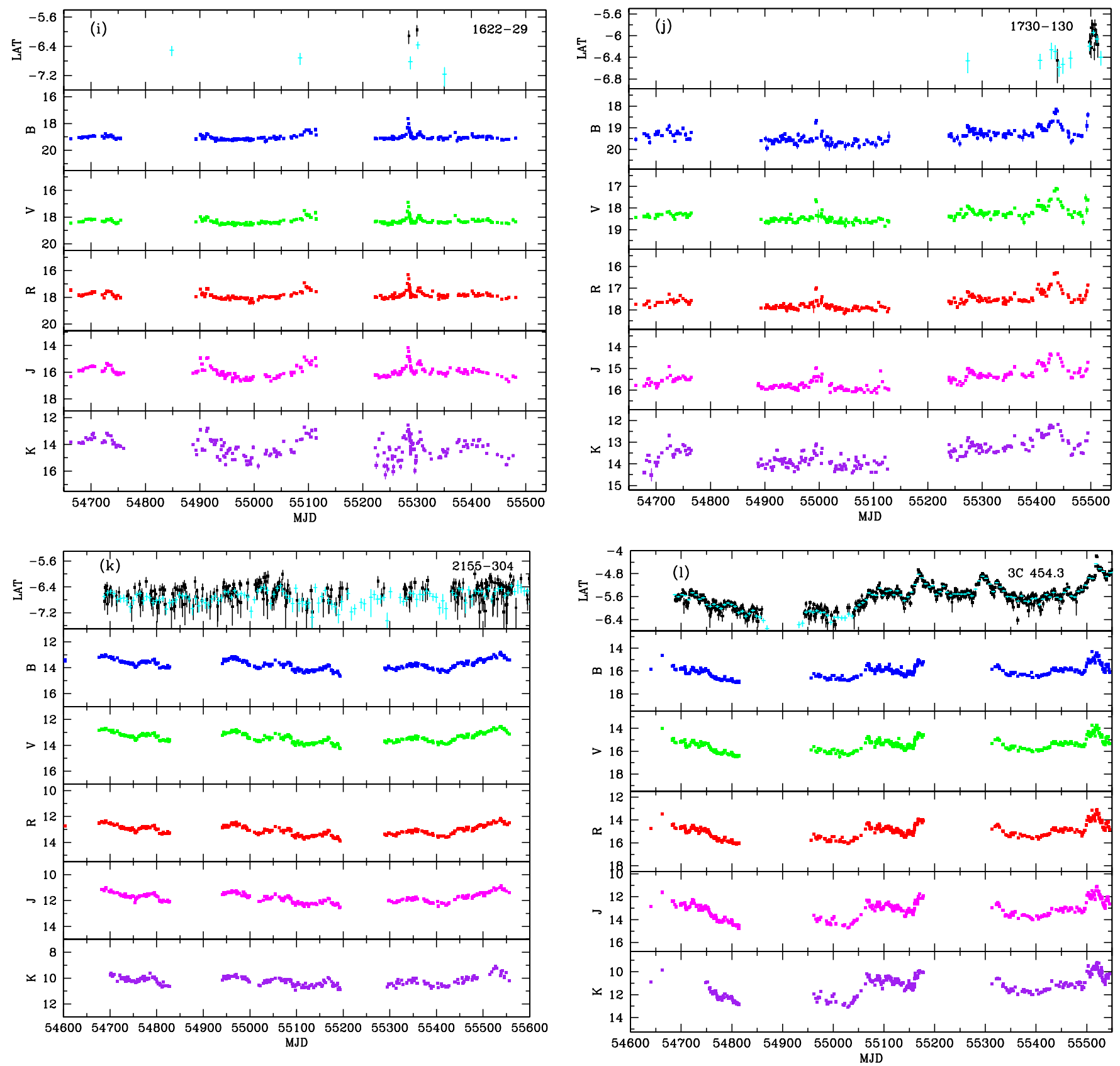

Figure 1. (Continued)

having weak emission lines, are likely to have weakly accreting or radiatively inefficient disks, so it is not surprising that they do not behave as the majority of our FSRQs. However, this is a small sample, which includes the unusual source AO 0235+164 in which broad emission lines have been observed, the strength of which calls into question its status as an LBL (Cohen et al. 1987; Nilsson et al. 1996). Nevertheless, the SED of AO $0235+164$ shows a strong and variable UV component in addition to the synchrotron and inverse-Compton peaks (Raiteri et al. 2008), which may explain its variability behavior observed here.

\section{COLOR-MAGNITUDE VARIATIONS}

We present in Figure 5 the relation between IR ( $J$-band) magnitude and optical-IR spectral shape of the low-energy peak of the SED (given by $B-J$ color, such that a larger value corresponds to a redder color). Overall, luminous blazars (FSRQs) are redder when brighter and bluer when fainter. A typical example is shown in Figure 5(i) (PKS 1622-29). As the source varies in brightness, the color changes along a narrow locus in the color-magnitude plane, such that when the source is brighter, the color gets redder. Conversely, when the source is fainter, the color gets bluer (although the SED remains synchrotron-dominated and therefore red). This behavior has been seen previously in this source by Osterman Meyer et al. (2008). This "flattening" of the SED in faint states suggests that a strong, blue accretion disk component is mixed with redder jet emission, which may only be visible as a typical "blue bump" in extremely faint states (e.g., as observed in 3C 454.3 by Raiteri et al. 2007). Most of our FSRQs (with exceptions noted below) follow this redder-when-brighter track, consistent with FSRQs having luminous accretion disks, as also evidenced by their typically strong emission lines. 


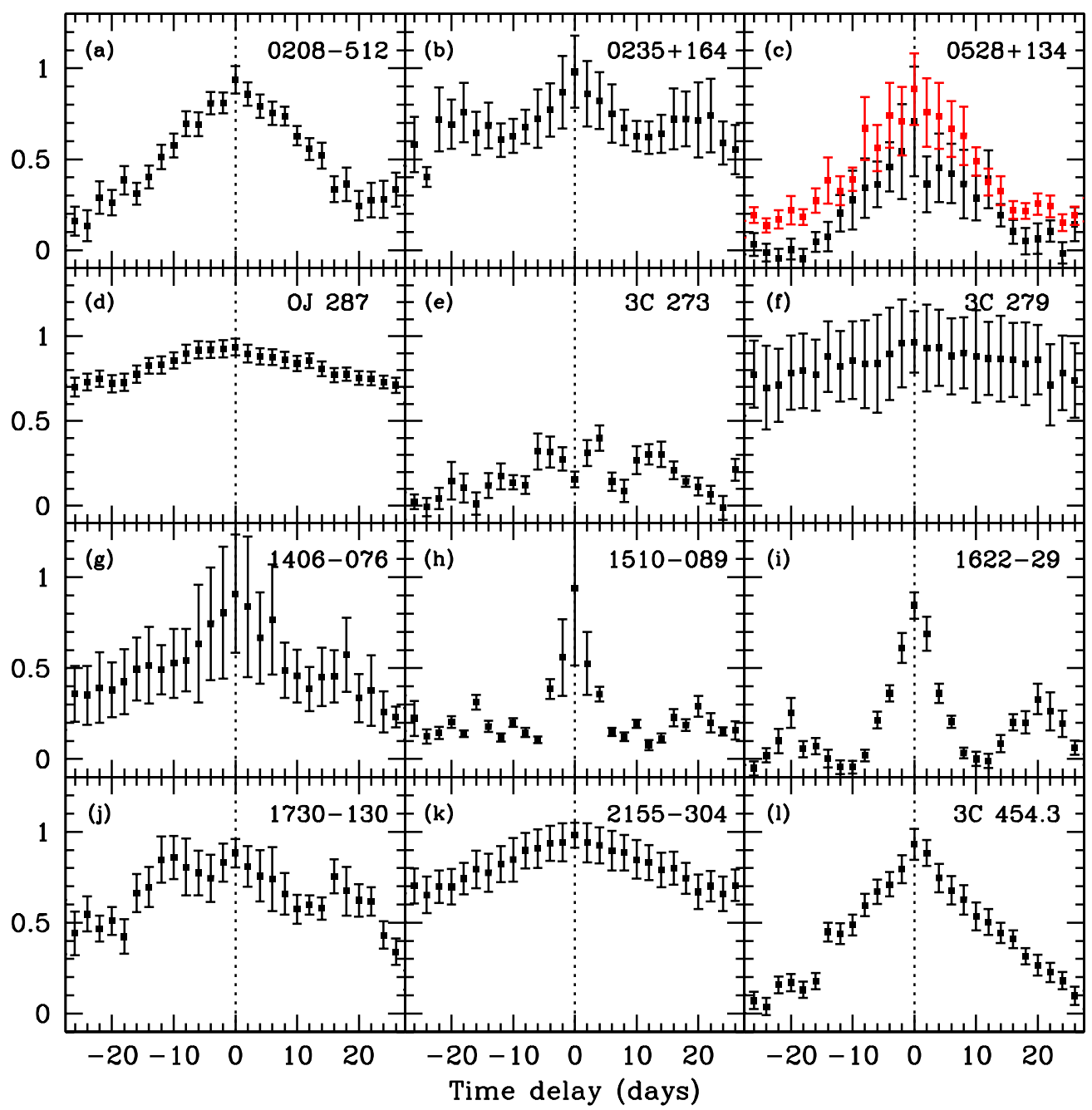

Figure 2. Discrete correlation functions (DCF) for $B$ vs. $J$-band light curves shown in Figure 1. With the exception of the minimally varying quasar $3 C 273$, all DCFs show a maximum at zero lag. Panel (c) also shows the $R$ vs. $J$ DCF for PKS $0528+134$ (in red). It is the only source for which the $R$ vs. $J$ DCF has a significantly different shape than $B-J$, although it too peaks at zero lag.

(A color version of this figure is available in the online journal.)

Table 2

Fractional Variability Amplitude (As Defined in Equation (1)) for Observed IR, Optical, and Gamma-rays

\begin{tabular}{llcccccc}
\hline \hline Name & Class & $K$ & $J$ & $R$ & $V$ & $B$ & $\gamma$ \\
\hline PKS 0208-512 & FSRQ & 0.48 & 0.52 & 0.45 & 0.40 & 0.43 & $\ldots$ \\
AO 0235+16 & LBL & 1.10 & 1.22 & 1.33 & 1.31 & 1.33 & 0.38 \\
PKS 0528+134 & FSRQ & $\ldots$ & 0.53 & 0.28 & 0.24 & 0.35 & $\ldots$ \\
OJ 287 & LBL & 0.45 & 0.37 & 0.43 & 0.45 & 0.46 & $\ldots$ \\
3C 273 & FSRQ & $\ldots$ & 0.13 & 0.041 & 0.044 & 0.042 & 0.77 \\
3C 279 & FSRQ & 0.49 & 0.79 & 0.82 & 0.87 & 0.86 & 0.38 \\
PKS 1406-076 & FSRQ & $\ldots$ & 0.61 & 0.48 & 0.49 & 0.46 & $\ldots$ \\
PKS 1510-08 & FSRQ & $\ldots$ & 1.04 & 0.94 & 0.94 & 0.83 & 0.56 \\
PKS 1622-29 & FSRQ & 0.68 & 0.54 & 0.36 & 0.29 & 0.27 & $\ldots$ \\
PKS 1730-130 & FSRQ & 0.58 & 0.47 & 0.37 & 0.34 & 0.35 & $\ldots$ \\
PKS 2155-304 & HBL & 0.28 & 0.33 & 0.33 & 0.33 & 0.33 & 0.25 \\
3C 454.3 & FSRQ & 0.68 & 0.58 & 0.51 & 0.47 & 0.44 & 1.40 \\
\hline
\end{tabular}

Note. The gamma-ray values are listed only for blazars bright enough to be detected in daily Fermi/LAT averages.

Although the FSRQs mostly show redder-when-brighter trends (e.g., Rani et al. 2010; Gu et al. 2006), they can also show more complicated behavior. For example, the 2010 May flare in PKS 1510-089 (indicated by arrows in Figure 5(h)) was essentially achromatic and, unlike its other flares, had a much larger amplitude than the associated gamma-ray flare (cf. Figure 1(h)). Such anomalous flares indicate the interplay of distinct components in the source; in the case of PKS 1510-089, the sharp achromatic flare could be caused by a plasma blob hitting a transverse shock in the jet (Marscher et al. 2010). (More speculatively, achromatic flares could be caused by gravitational microlensing in the halo of our galaxy, as in the MACHO experiment (Alcock et al. 2000), since these blazar jets are effectively point sources, but the probability of such an event should be vanishingly small.) Similarly, the 2009 December flare of 3C 454.3 (Figure 5(1)) moved off the standard red-bright track, although it was not as obviously achromatic. Such deviations have been seen, for example, in the 2007 December flare reported by Zhai et al. (2011). Even the relatively non-varying 3C 273 still shows a redder-when-brighter track in the color-magnitude plane, although we note that Dai et al. (2009) have found the opposite trend during a two-year observation from 2003-2005.

In contrast to FSRQs, the lower luminosity BL Lac objects do not show the same color-magnitude trend, consistent with BL Lac objects having weak accretion disks. The HBL PKS 2155304 is shown in Figure 5(k); its variations are more random 

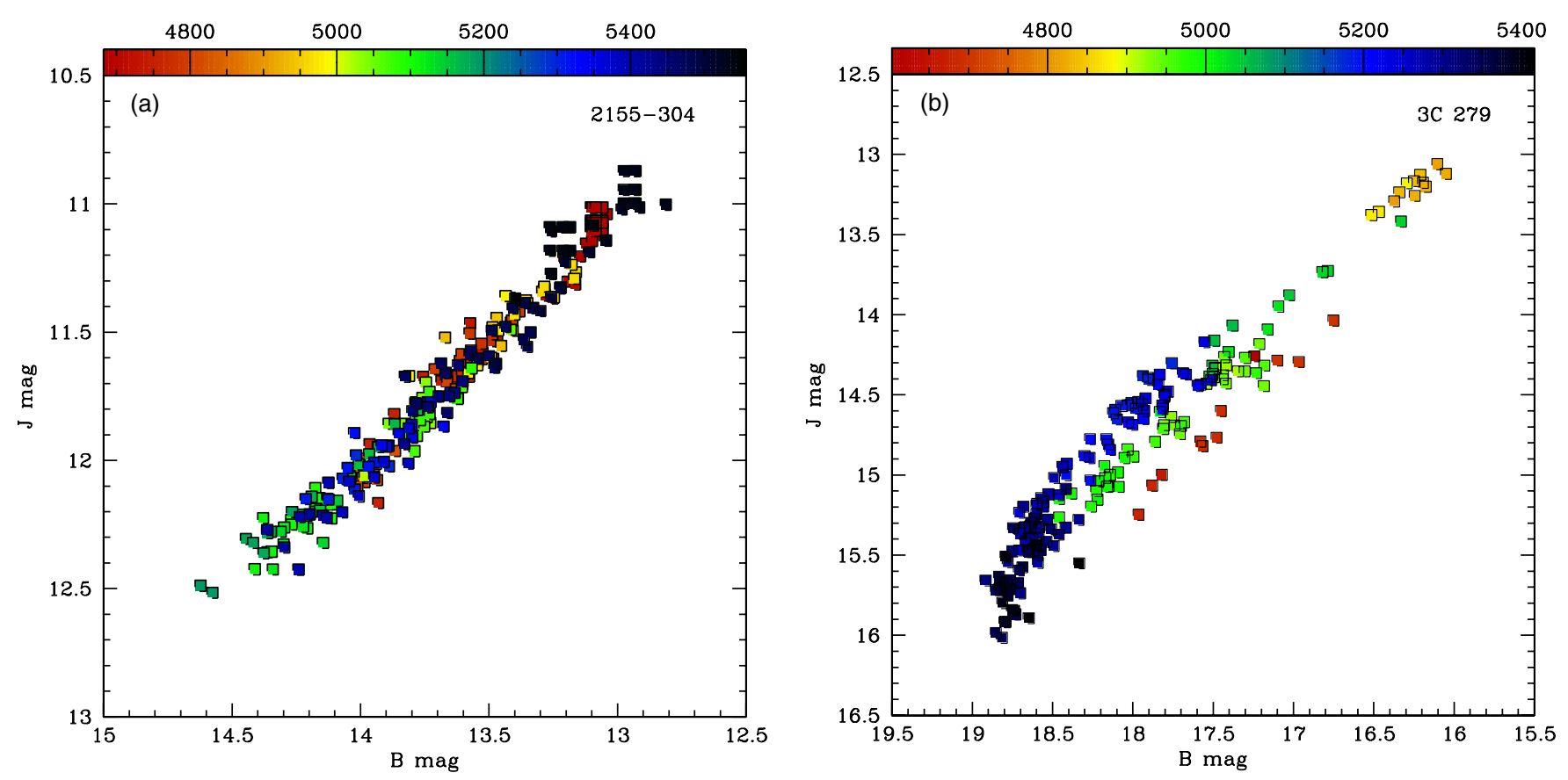

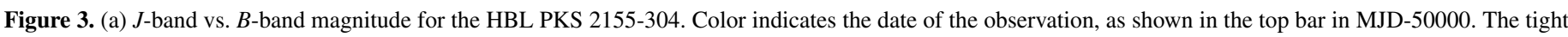

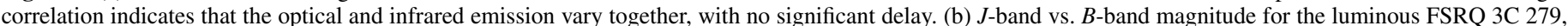

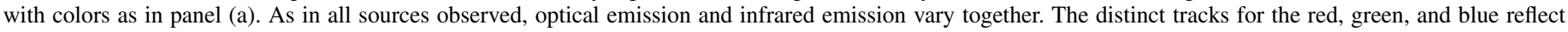
changes optical/IR spectral shape over time (cf. Figure 5(b)).

(A color version of this figure is available in the online journal.)

with respect to color, and smaller in amplitude. Some studies at short timescales (hours to days) have shown a bluer-whenbrighter trend in this source (Paltani et al. 1997; Dolcini et al. 2007). Such short-timescale trends could be observed in our data, but the overall trend over several years reveals no strong correlation between color and brightness. This suggests that there is no strong persistent underlying component of the SED of PKS 2155-304.

Somewhat more complicated is the color behavior of the LBL OJ 287 (Figure 5(d)), which shows some redder-when-brighter changes but also variations that are bluer-when-brighter, and still other color changes at fixed magnitude. Like the HBL PKS 2155-304, OJ 287 has a range of $\sim 2 \mathrm{mag}$ in brightness, and no clear color-magnitude relation as seen in the FSRQs. However, OJ 287 does show structure within color-magnitude space. OJ 287 is known for a striking series of double-peaked outbursts occurring approximately every 12 years over the last century (Sillanpaa et al. 1988; Lehto \& Valtonen 1996). Our observing period begins after the last double outburst in 2005-2007. We find that OJ 287 is brighter and more variable post-burst than it was between the 2005 and 2007 peaks. As it was during the double-peaked bursts, OJ 287 remained highly polarized during the early 2009 observing season (see Figure 1 of Villforth et al. 2010), with a strong, stably, underlying optically polarized core. Villforth et al. also found an overall bluer-when-brighter color trend over the time period 2005-2009. Additionally, observations in 1993-1994 showed constant optical colors over a range in optical flux (Sillanpaa et al. 1996; Hagen-Thorn et al. 1998). They suggest that these changes in spectral shape are due to injections of high energy electrons into the jet emitting region or "wobbling" of the jet, possibly related to induced activity from the putative binary (see also Zheng et al. 2008, who additionally predict a periodicity in spectral index related to the putative binary.) Alternatively, it may be that the accretion disk component in
OJ 287 is variable and comparable in magnitude to the jet component. This is suggested by analogy to X-ray binaries, in which this complicated color-magnitude behavior is a known phenomenon, and hysteresis in the X-ray flux-spectral index plane has been interpreted in terms of accretion state changes (Smith et al. 2007; Maccarone \& Coppi 2003). (Due to their hotter accretion disks, the thermal peak in X-ray binaries lies in the soft X-ray, compared with the rest-frame UV for LBLs and FSRQs.) On the other hand, hysteresis behavior has also been reported for the HBL PKS 2155-304 (Kataoka et al. 2000), in which the X-rays come from the jet and no disk has been detected. This hypothesis can be tested by observing changes in emission lines; if the disk is varying, the lines should respond in intensity. On balance, it is not obvious what causes the unusual color-magnitude trends in OJ 287.

Still more anomalous behavior is exhibited by AO $0235+164$ (Figure 5(b)). At the beginning of our monitoring program, for example, increasing intensity was characterized by bluer (not redder) colors. This was also when AO 0235+164 was detected by Fermi in the daily light curve (i.e., it was gamma ray-bright, cf. Figure 1(b)). Later, when the source was much fainter in gamma rays, the color-magnitude trend reverted to the usual redder-when-brighter relation.

AO $0235+164$ is an unusually situated source. An AGN sits $2^{\prime \prime}$ to the south of the blazar as well as an intervening galaxy at $z=0.525$ (Yanny et al. 1989; Burbidge et al. 1996; Nilsson et al. 1996), which we do not resolve in our imaging. The AGN has a fairly blue spectrum $(B=21.4, V=20.9, R=20.5)$, which has not been observed to vary strongly (Raiteri et al. 2005). The extinction due to the intervening galaxy is nearly a magnitude greater than the Galactic extinction $\left(A_{B}=1.268\right.$ versus 0.341 , Junkkarinen et al. 2004), resulting in a very intrinsically red spectrum. We removed the contribution from this AGN in the $B$ band before plotting the color-magnitude relation in Figure 5(b), so the bluer-when-fainter behavior of $\mathrm{AO} 0235+164$ is not due to 


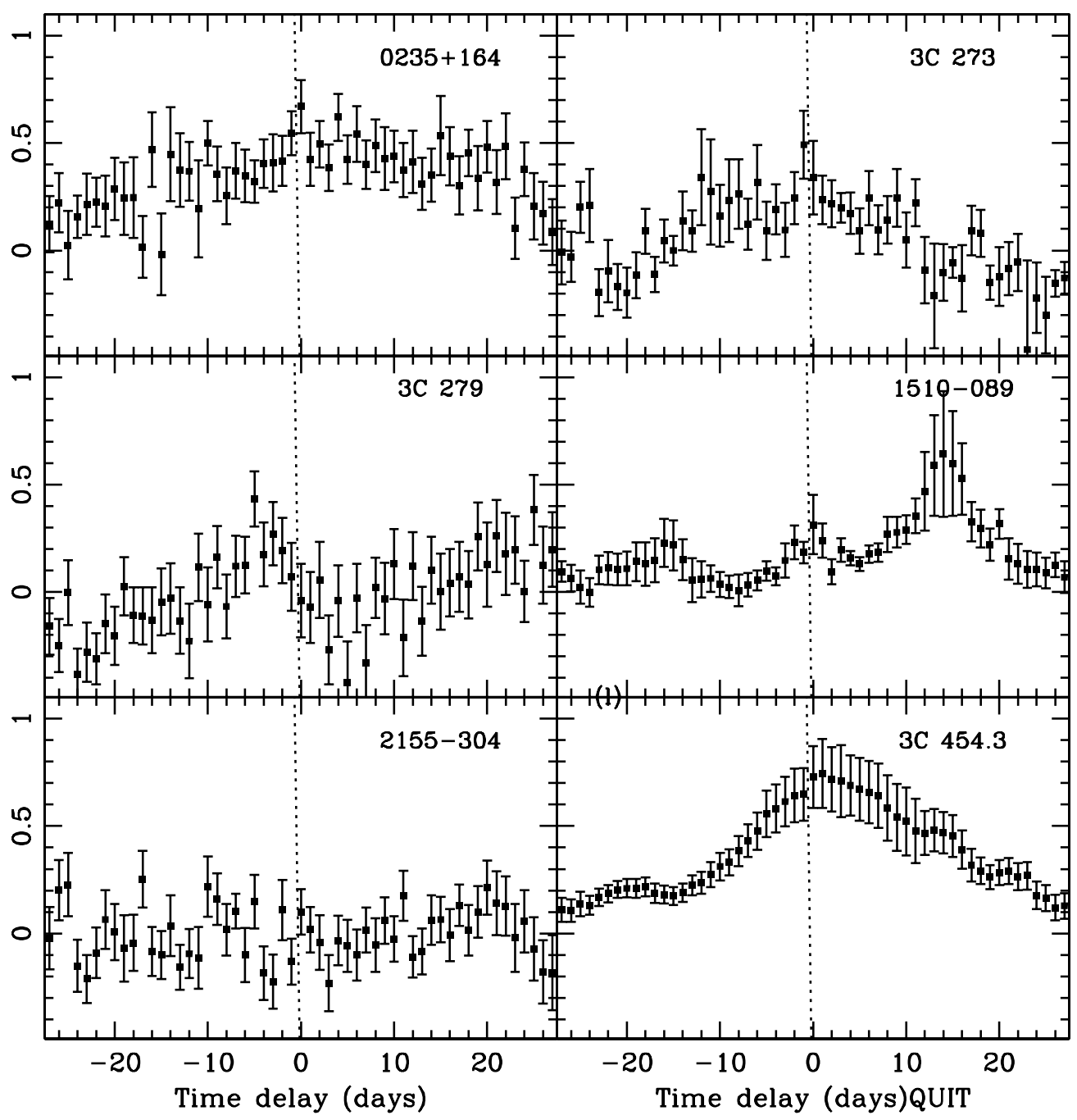

Figure 4. Discrete correlation functions (DCF) for $\gamma-J$-band light curves shown in Figure 1. A positive time delay corresponds to $J$ band lagging the gamma-rays. In several sources (3C 454.3, most clearly) the emission is well correlated with little or no lag. In others, the DCF is flat, suggesting no strong correlation (PKS 2155-304). In PKS 1510-089, the gamma-rays appear to lead the $J$ band by a couple of weeks.

the underlying blue colors of the nearby AGN. Complex spectral behavior has been observed in this source by Raiteri et al. (2006), who find significant variation in spectral index over more than a decade and a half. Over this timescale; however, they do not note any particular correlation with brightness.

If the observed optical-IR emission is a combination of jet and accretion disk, and if the gamma rays are produced by inverse Compton scattering of disk or line photons, then the two trends can be explained self-consistently. When the jet becomes brighter, the color of the combined emission gets redder since the jet synchrotron emission is intrinsically redder than that of the blue accretion disk. Alternatively, if the jet is constant and the disk emission increases, then the summed emission becomes bluer. Moreover, an increase in disk emission would be accompanied by a strong increase in gamma rays, due to the increased scattering of disk/line photons, hence the association of a bright gamma-ray state with the bluer-when-brighter trend. In addition, when the jet is quite bright ( $J \gtrsim 14$ in this case), further brightening of the jet may be due to emission from an even higher energy population of electrons; this would also result in a bluer color. 3C 279 (Figure 5(b)) has a very similar (i.e., hybrid) color-magnitude diagram, roughly achromatic in the optical when bright in gamma rays but much fainter in gamma rays when it moves along the more usual FSRQ bluerwhen-fainter trend.

In the examples of AO 0235+16 (Figure 5(b)) and 3C 279 (Figure 5(f)), different loci in the optical/IR color-magnitude diagram are associated with different gamma-ray intensities. This bimodal behavior has some similarity to the Galactic $\mathrm{X}$-ray binaries. For example, the X-ray binary GX 3394 has been observed to change from a bluer-when-brighter state (associated with the high/soft X-ray state) to a redderwhen-brighter state (associated with the hard X-ray state-see Buxton et al. 2012). X-ray binary emission is a combination of soft X-rays from a disk and hard X-rays from a power-law component (likely from a hot corona). In the canonical "high-soft" state, the X-ray spectrum is disk-dominated and hence, softer, but the intensity is higher, while in the canonical "low-hard" state, the intensity is lower but the spectrum is dominated by the power-law component, and hence, is harder (Remillard \& McClintock 2006). There is a third so-called "very high state" in which the XRB spectrum is dominated by the power-law component at a very high intensity. For these anomalous FSRQs, the gamma-ray bright times may correspond to the "very high state," in which case the optical emission will also be very bright and will get bluer when brighter because it is totally jet dominated. 

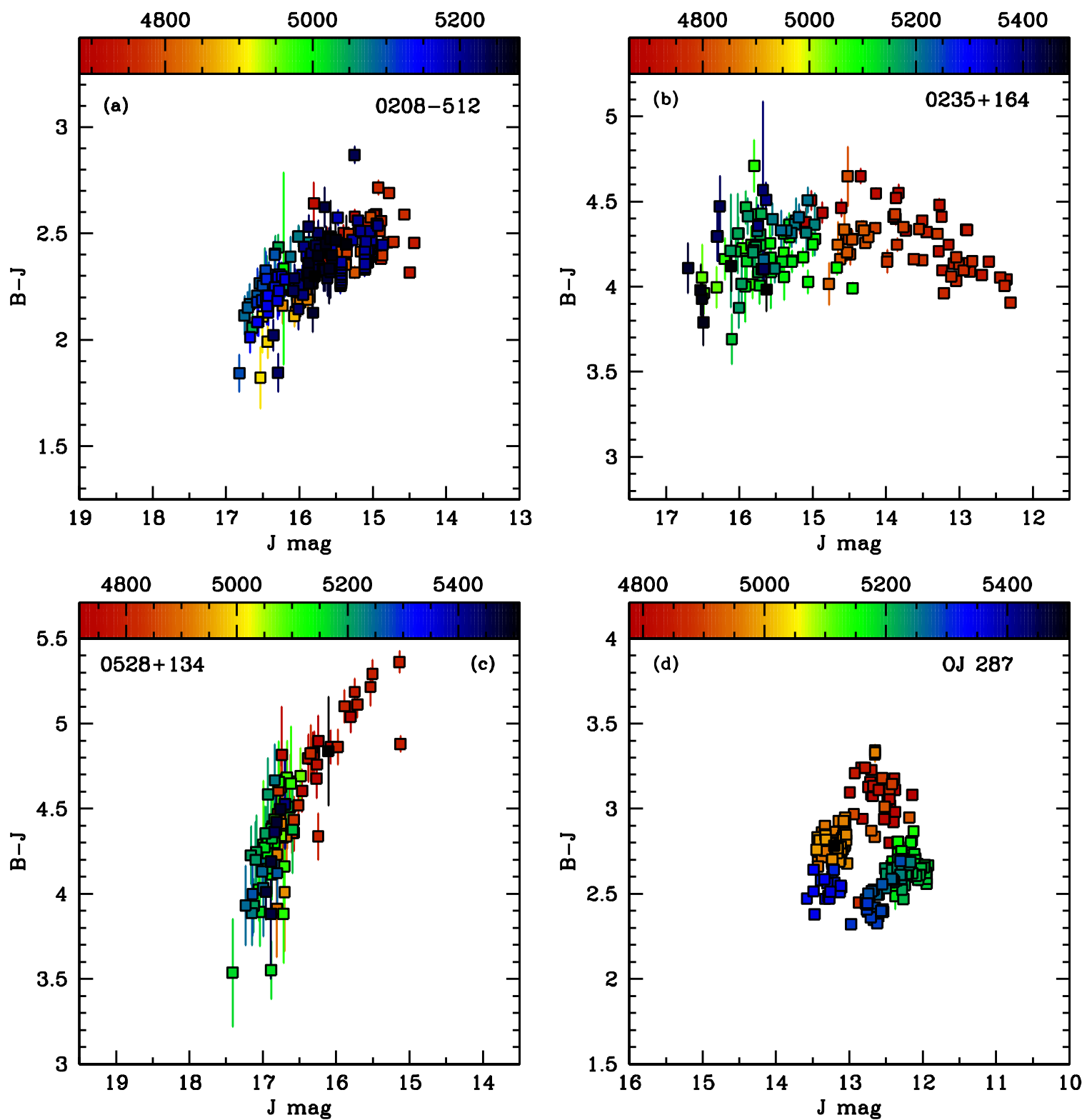

Figure 5. (a-d) $(B-J)$ color vs. $J$-band magnitude for FSRQs PKS 0208-512 and PKS 0528+134, and low-frequency peaked BL Lac objects AO 0235+164 and OJ 287. Color indicates the date of the observation, as shown in the top bar in MJD-50000. The FSRQs show an overall tendency to become bluer when fainter, which suggests the presence of a steady blue accretion disk component underlying the more variable jet emission. The two LBLs show more complicated behavior: AO $0235+164$ shows some redder-when-brighter trend (blue-black points) but at early times (red-orange points) shows almost the opposite trend, getting bluer when brighter. During this bluer-when-brighter period, AO 0235+164 was very bright in gamma-rays and was easily detected by Fermi on daily timescales. When AO 0235+164 became fainter in the optical and was not detected in the daily LAT light curve, it shifted to the "normal" (for FSRQs) bluer-when-fainter trend. OJ 287 tends to move around on a circular locus in color-magnitude space where trends with brightness (including gamma-ray intensity) are not as easily definable. (e-h) $(B-J)$ color vs. $J$-band magnitude for FSRQs 3C 273, 3C 279, PKS 1406-076, and PKS 1510-089. Color indicates the date of the observation, as shown in the top bar in MJD-50000. All of these FSRQs show the overall redder-when-brighter (bluer-when-fainter) trend, consistent with a steady blue accretion disk component underlying the jet emission. Individual flares can behave differently; for example, the flare in PKS 1510-089 in 2010 May is achromatic (arrows indicate the 4 green points tracing a horizontal trend). The quasar 3C 279 shows compound behavior similar to AO 0235+164 (panel 5(b)), with the red-light green points tracing a horizontal loop in the color-magnitude figure before and just up to a period of bright gamma-ray activity (cf. Figure 1(f)). (i-1) $(B-J)$ color vs. $J$-band magnitude for FSRQs PKS 1622-29, PKS 1730-130, and 3C 454.3, and the HBL PKS 2155-304. Color indicates the date of the observation, as shown in the top bar in MJD-50000. The FSRQs show the redder-when-brighter trend. However, for the HBL, the brightness and spectral changes are relatively small, and there is no trend of color with magnitude. This may be because the HBL both lacks a luminous accretion disk and the optical jet emission in this object is below the synchrotron peak, thus optically thick and not highly variable.

(A color version of this figure is available in the online journal.)

\section{THE TIME-DEPENDENT SPECTRAL ENERGY DISTRIBUTION}

Figure 6 shows the variable broadband SED for 3C 454, with quasi-simultaneous data (within hours) for UT 2009 December 3 (blue) and UT 2009 December 4 (red), when the source was near the peak of its large 2009 December flare. A representative
SED for a non-flaring state is shown for UT 2009 August 12 when the source was relatively faint (black). The X-ray (Swift $\mathrm{XRT}$ and BAT) and gamma-ray (Fermi) data points shown are taken from the analysis in the paper of Bonnoli et al. (2011). The NIR-optical points shown are the SMARTS monitoring data presented in this paper, dereddened using the extinction relations in Cardelli et al. (1989), with the value for $A_{\mathrm{B}}$ given by 

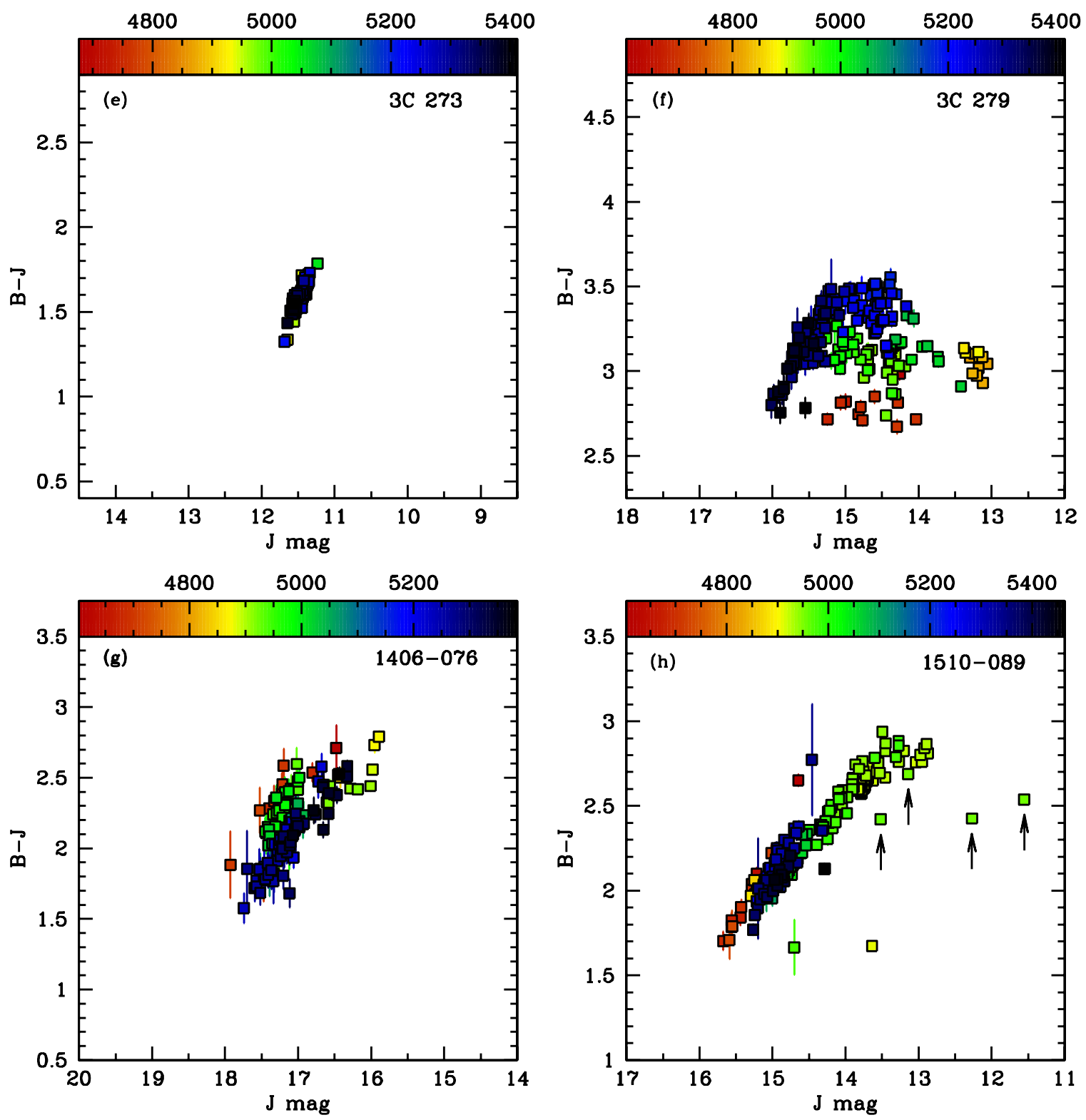

Figure 5. (Continued)

Schlegel et al. (1998). Magnitudes were converted into flux densities using the zero-point fluxes given by Bessell et al. (1998) and Beckwith et al. (1976) The observing conditions were photometric with good seeing on the nights shown in Figure 6. The near-IR part of the spectrum shows a significant change between the two nights, turning over between $J$ and $K$ bands. We searched the light curve of 3C 454.3 for similar behavior and found similar turnovers in the NIR SED on 12 nights, generally also with good observing conditions. This flattening of the SED occurred in faint states as well as bright states.

The gamma-ray (inverse Compton) and optical-NIR spectra (presumably the high-energy end of the synchrotron emission component) both varied significantly during the 2009 December 3-4 flare, but in opposite senses. That is, the gamma-ray spectrum flattened and declined slightly while the optical/IR steepened and increased in flux. This definitely points to complex physical changes beyond a simple electron acceleration event. Note that the SMARTS data are extended enough in energy coverage that one can see that the synchrotron spectrum is not consistent with a power law and that the synchrotron emission peak likely moved. On the other hand, if one ignored the NIR ( $J, K$ band) points, then one might conclude from the small spectral changes in the optical and gamma-rays that the underlying electron energy distribution and source parameters were roughly similar. This was the conclusion of Bonnoli et al. (2011), who focused on fitting the X-rays and gamma-rays and only roughly matched the optical intensity of the source. Thus the ability of SMARTS to cover both optical and IR bandpasses constrains the physical interpretation in ways that gamma-ray or optical monitoring alone does not.

The SEDs on the two nights of the 2009 December flare were modeled using the one zone code of Coppi (1992). This model injects electrons with a power-law distribution in energy, turning over at the high energy end, giving effectively a maximum and minimum $\gamma$, as also used in the models of Ghisellini et al. (cf. Ghisellini et al. 2007). We assume a soft photon field with a black body temperature of $k T \sim 1-10 \mathrm{eV}$ that is isotropic in the jet frame. The electron distribution is convolved with the synchrotron spectrum and thermal photon field to produce 

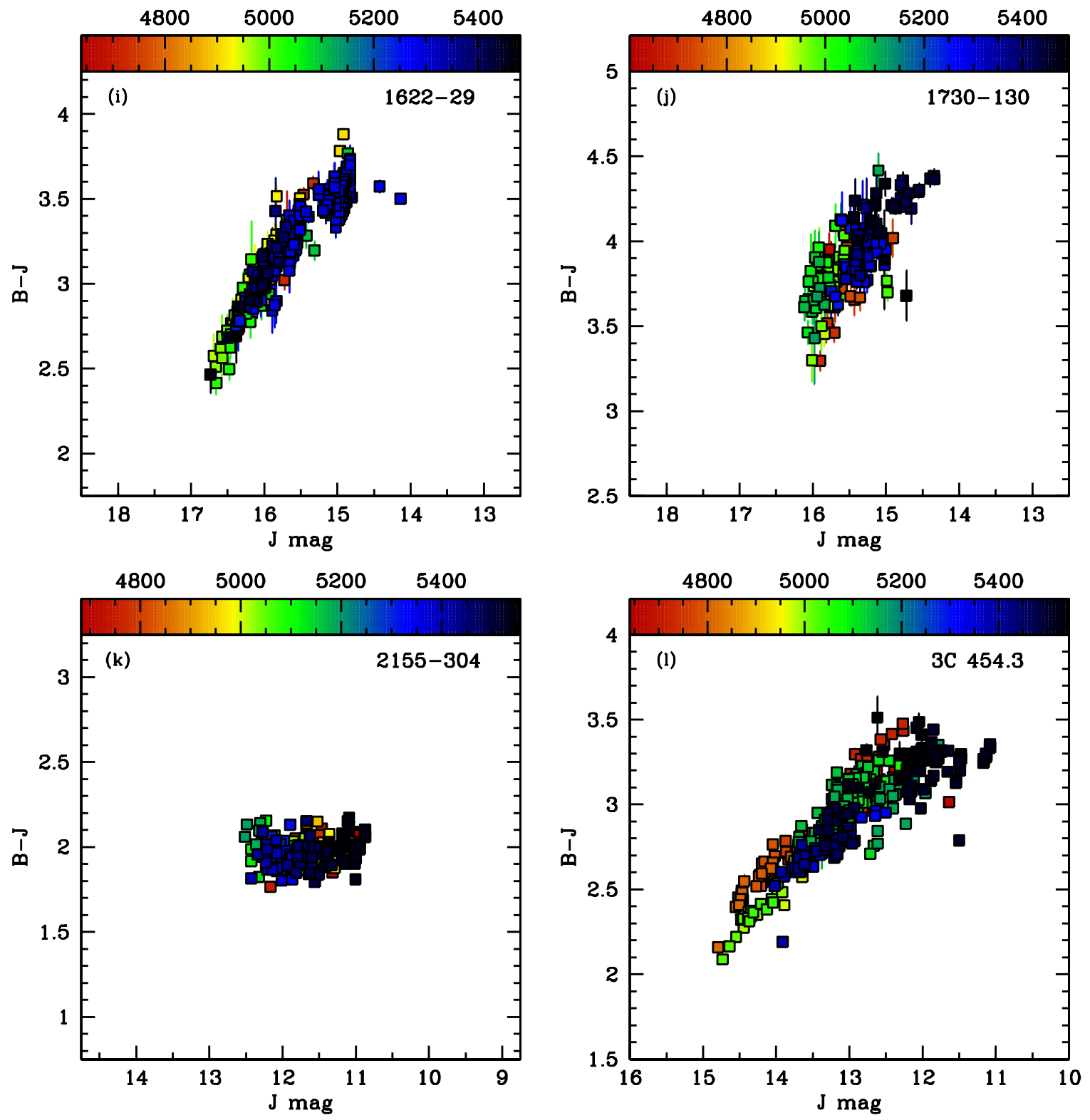

Figure 5. (Continued)

gamma-rays. The key difference between the Coppi (1992) code and others is that the electron spectrum changes through both synchrotron and Compton cooling. In the Klein-Nishina limit, Compton cooling becomes inefficient while synchrotron cooling is not affected, thus producing more power in the high-frequency end of the synchrotron radiation. This may be what is seen in the $J-K$ band peak.

If we assume the optical-NIR emission is produced in the same source region as the gamma-rays (which is not unreasonable as this is a very bright flare and the gammaray and optical variations are correlated), then our preliminary SED modeling of the NIR to gamma-ray data (shown by the solid red and blue curves in Figure 6) indicates that the source variations must be significant. For source parameters similar to Bonnoli et al. $\left(2011, R_{\text {source }}=\sim 10^{17} \mathrm{~cm}, \delta \sim 20, B \sim 1\right.$, $\gamma_{\text {peak }} \sim 500$ ), we find that the electrons responsible for the optical-NIR emission are likely higher in energy than those responsible for the gamma-ray emission, and that Klein-Nishina effects are thus important for them. This means that in order to produce a steeper yet higher amplitude synchrotron spectrum, the external photon field seen by the emitting electrons must have changed to include more lower-temperature photons than the BLR; see Moderski et al. (2005b) and Moderski et al. (2005a) for a discussion of the dependence of Klein-Nishina effects on the external photon field. Varying the ambient photon field alone, however, is not enough to explain all the observed spectral changes: In the context of traditional one-zone synchrotronCompton models, some combination of peak electron energy, the magnetic field, and the Doppler beaming factor must have changed by factors of two or more.

We note here that this is not a systematic exploration of parameter space, but merely a demonstration that the shortterm variations in the SED cannot be produced merely through changing the maximum value of $\gamma$, or the strength of the magnetic field alone. The change in model parameters has to be more complicated. Further exploration of multiwavelength spectral variability will be presented in a forthcoming paper (R. Chatterjee et al. 2012, in preparation). We further note that if our preliminary conclusions are correct, namely that gammaray and optical emission comes from somewhat different energy 


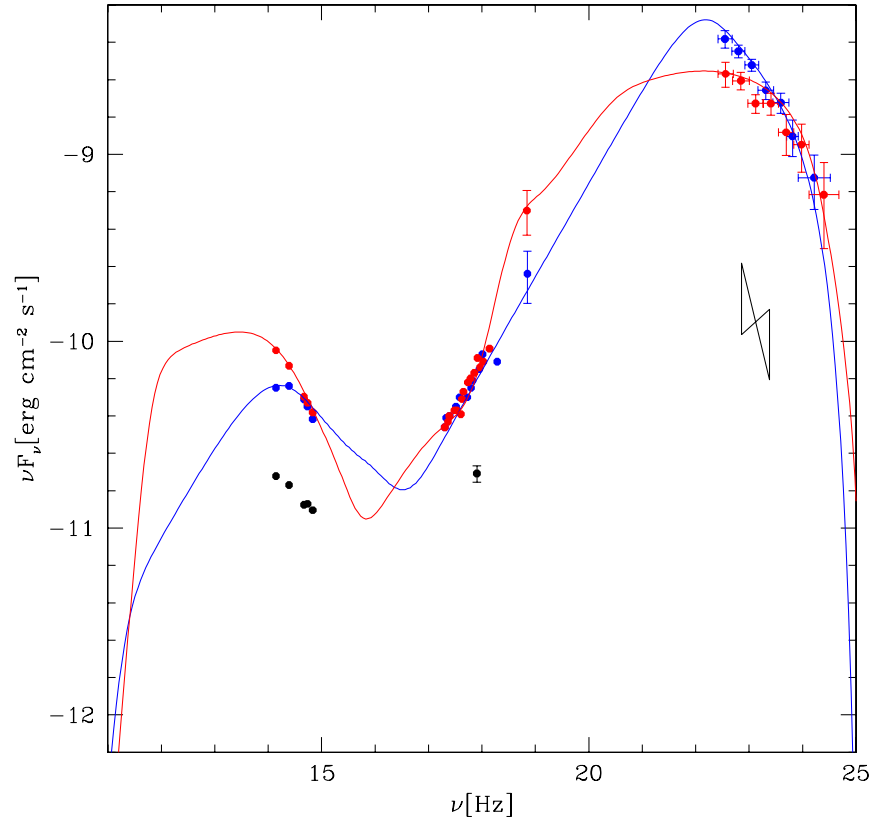

Figure 6. Spectral energy distribution of 3C 454.3 from data obtained during the bright flare on UT 2009 December 3 (blue points) and UT 2009 December 4 (red points, and in a low state on UT 2009 August 12 (black points). Blue and red lines are model fits to the entire dataset using the one-zone code of Coppi (1992). Error bars on the optical and NIR points are smaller than the plotting symbol. The infrared SMARTS data clearly shows a change in synchrotron peak energy between December 3 and 4; modeling this together with the changing gamma-ray peak and slope poses challenges for simple single-zone models. However, these simple fits are sufficient to indicate that Klein-Nishina effects are likely important and that the optical-IR is produced by electrons of higher energy than those producing the gamma rays.

(A color version of this figure is available in the online journal.)

electrons, and that the mapping from electron energy to synchrotron and Compton photon energy may change during the flare, then this might explain why we see general but not exact correlations between the variability in gamma-ray and lower energy bands. One-zone models may still have some relevance during bright flares, if one region of the jet dominates the overall emission. Finally, this implies that the lore that Fermi spectra do not change much during flares appears to be at best a coincidence in 3C 454.3: The underlying electron and target photon spectra probably change significantly.

\section{CONCLUSIONS}

Systematic monitoring of southern blazars with the SMARTS optical+infrared ANDICAM has generated BVRJK light curves for a dozen blazars, all of which were contemporaneously observed with the Fermi Gamma-Ray satellite. All of the SMARTS blazar photometry is available on a publicly accessible Web site, http://www.astro.yale.edu/smarts/fermi. This paper reported on the 12 blazars that have well defined optical/IR light curves over the first 2 years of the Yale/SMARTS blazar monitoring project, which commenced in 2008 August.

Not all 12 blazars are significantly detected in gamma-rays on $\sim$ day timescales. For the first two years of data (through 2010 July), six SMARTS-monitored blazars were bright enough to be well detected in one-day averages at gamma-ray energies: the FSRQs 3C 454.3, 3C 273, 3C 279, and PKS 1510-089; the LBL AO 0235+164; and the HBL PKS 2155-304. Detailed multiwavelength analysis was presented for these six.

We find the optical/IR and gamma-ray light curves for these six southern blazars are generally well correlated, with lags of less than 1 day for most FSRQs (roughly the sampling interval for the SMARTS and Fermi data). This strongly supports leptonic models in which ambient photons-perhaps from the accretion disk or broad emission-line clouds - are upscattered to gamma-ray energies by synchrotron-emitting electrons in the relativistic blazar jet. Hadronic models, in which the gammarays come from energetic protons that ultimately decay into synchrotron-emitting electrons, do not have the same natural explanation for correlated optical/IR and gamma-ray variability.

The multiwavelength variability for the six blazars shows two types of behavior. In luminous blazars like FSRQs, the amplitude of optical/IR variability decreases toward shorter wavelengths, as if that wavelength range had a significant underlying contribution from a more slowly varying thermal disk peaking in the UV. In BL Lac objects, the optical/IR variability is similar in different bands, consistent with little or no contribution from a (radiatively inefficient) disk.

Color-magnitude variations in these six blazars can be explained in part by highly variable jet emission mixed with slower varying disk emission. Gamma-ray flares can result from particle acceleration in the jet or from secular increases in disk/line emission, hence the somewhat complicated trajectories of blazars in color-magnitude space. Analogous multiple-state behavior has been observed in Galactic X-ray binaries. Meanwhile, individual jet flares in a given source can also follow different color-color tracks because of, for example, standing shocks in different parts of the jet.

The best-studied blazar, the FSRQ 3C 454.3, has had a strong flare in each year of SMARTS-Fermi monitoring. Its optical/IR and gamma-ray light curves are well correlated, with no measurable lag longer than one day. Its broadband SED in the bright state is well fit by an external Compton model; in its faint state, the disk contributes a larger fraction of the light and the SED is flatter. Day-to-day changes are difficult to explain with a simple one-zone model, however, unless there are large changes (factors of two or more) in Doppler factor, electron energy, and/or magnetic field. More generally, our simple analysis of the 3C 454.3 SED suggests that optical/IR radiation comes from slightly higher energy electrons than the $\mathrm{GeV}$ gamma rays, and that Klein-Nishina effects are important in shaping both SED components.

SMARTS monitoring of southern blazars continues, with the goal of producing additional rich multiwavelength data sets that will further advance our understanding of blazar emission mechanisms and energy transport. In more than three years of monitoring several dozen blazars, only six sources-in three different classes-have produced multiwavelength data of sufficient quality for detailed analysis. Only some of these showed distinct flares over this period. The rarity of blazars being bright while visible from the ground (often there have been dramatic gamma-ray flares while the sources had not yet risen in the night sky) suggests that continued dedicated optical-infrared monitoring is essential during the lifetime of the Fermi GammaRay Observatory.

SMARTS observations of LAT-monitored blazars are supported by Fermi GI grant 011283 and by Yale University. C.D.B., M.M.B., and the SMARTS $1.3 \mathrm{~m}$ observing queue receive support from NSF grant AST-0707627. R.C. is supported by Fermi GI grant NNX09AR92G. G.F. is supported by Fermi GI grant NNX10A042G. J.I. is supported by the NASA Harriet Jenkins Fellowship program and NSF Graduate Research Fellowship under Grant No. DGE-0644492. This research has 


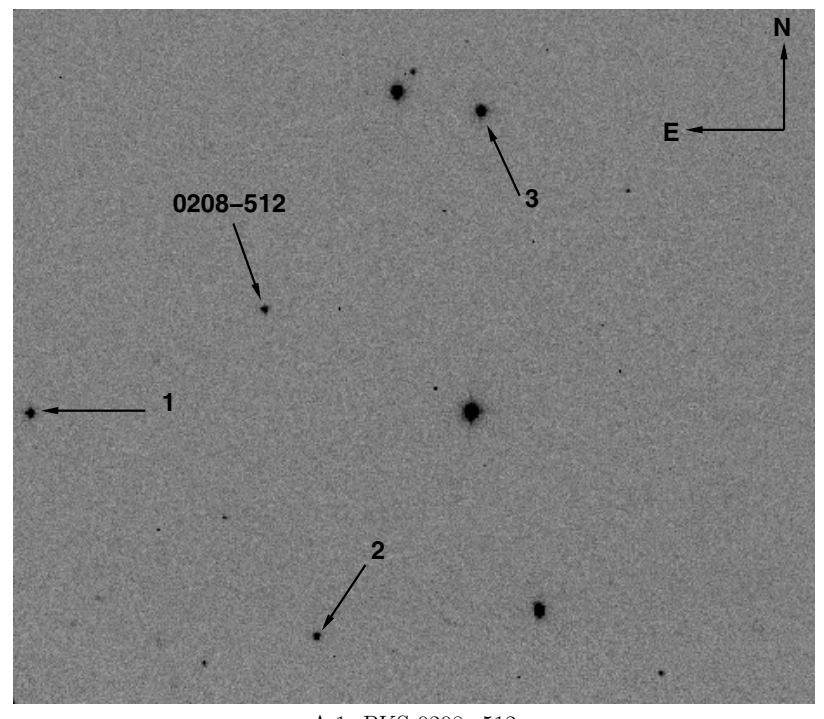

A.1: PKS 0208-512.

Figure 7. (A.1) PKS 0208-512, (A.2) AO 0235+164, (A.3) PKS 0528+134, (A.4) OJ 287, (A.5) 3C 273, (A.6) 3C 279, (A.7) PKS 1406-076, (A.8) PKS 1510-089, (A.9) PKS 1622-297, (A.10) PKS 1730-130, (A.11) PKS 2155-304, and (A.12) 3C 454.3.

(An extended version of this figure is available in the online journal.)

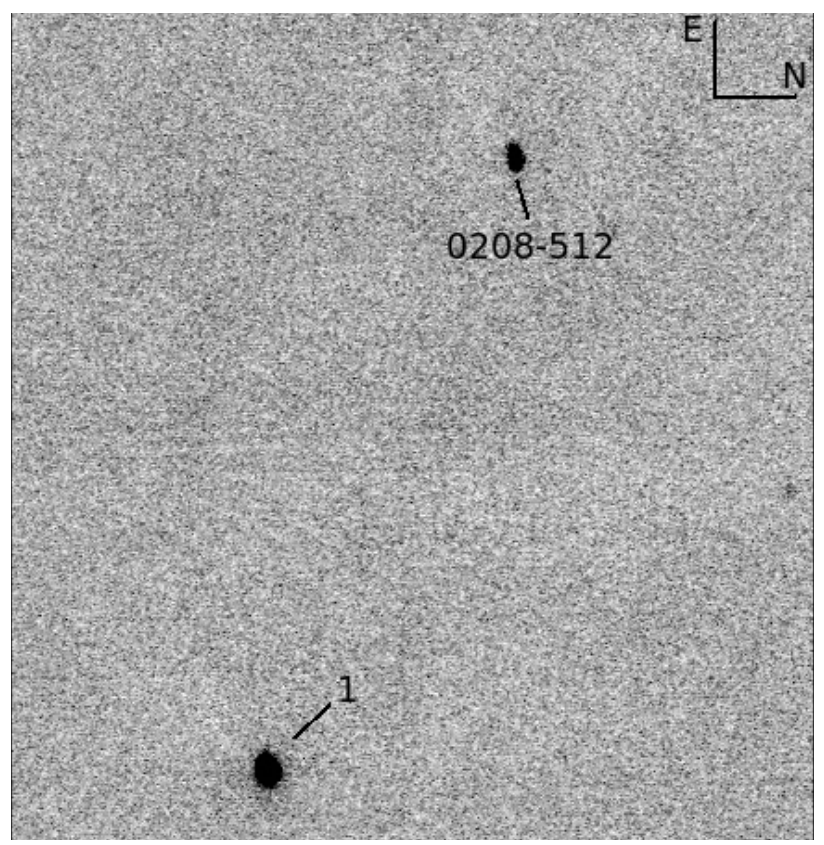

B.1: PKS 0208-512

Figure 8. (B.1) PKS 0208-512, (B.2) AO 0235+164, (B.3) PKS 0528+134, (B.4) OJ 287, (B.5) 3C 273, (B.6) 3C 279, (B.7) PKS 1406-076, (B.8) PKS 1510-089, (B.9) PKS 1622-297, (B.10) PKS 1730-130, (B.11) PKS 2155-304, and (B.12) 3C 454.3.

(An extended version of this figure is available in the online journal.)

made use of the NASA/IPAC Infrared Science Archive, which is operated by the Jet Propulsion Laboratory, California Institute of Technology, under contract with the National Aeronautics and Space Administration.

\section{APPENDIX A}

\section{OPTICAL FINDING CHARTS}

We present optical finding charts for each of the 12 SMARTSmonitored blazars discussed in this paper. All figures are $V$-band
Table 3

Comparison Star Optical Magnitudes

\begin{tabular}{|c|c|c|c|c|}
\hline Source & Star & $B$ & $V$ & $R$ \\
\hline \multirow[t]{3}{*}{ PKS 0208-512 } & 1 & $16.43 \pm 0.03$ & $15.72 \pm 0.03$ & $15.27 \pm 0.04$ \\
\hline & 2 & $16.90 \pm 0.04$ & $16.24 \pm 0.04$ & $15.79 \pm 0.03$ \\
\hline & 3 & $15.43 \pm 0.04$ & $14.69 \pm 0.03$ & $14.29 \pm 0.03$ \\
\hline \multirow[t]{5}{*}{ AO $0235+164$} & 1 & $13.61 \pm 0.03$ & $13.05 \pm 0.03$ & $12.60 \pm 0.02$ \\
\hline & 2 & $13.55 \pm 0.04$ & $12.75 \pm 0.03$ & $12.26 \pm 0.03$ \\
\hline & 3 & $13.72 \pm 0.04$ & $12.98 \pm 0.03$ & $12.55 \pm 0.03$ \\
\hline & 4 & $14.70 \pm 0.04$ & $14.05 \pm 0.03$ & $13.64 \pm 0.03$ \\
\hline & 5 & $15.74 \pm 0.03$ & $14.80 \pm 0.03$ & $14.22 \pm 0.03$ \\
\hline \multirow{2}{*}{ PKS 0528+134 } & 1 & $15.90 \pm 0.05$ & $14.79 \pm 0.03$ & $13.99 \pm 0.05$ \\
\hline & 2 & $16.81 \pm 0.03$ & $15.80 \pm 0.03$ & $15.09 \pm 0.03$ \\
\hline \multirow[t]{4}{*}{ OJ 287} & 1 & $15.03 \pm 0.05$ & $14.25 \pm 0.04$ & $13.69 \pm 0.05$ \\
\hline & 2 & $15.18 \pm 0.04$ & $14.65 \pm 0.04$ & $14.26 \pm 0.04$ \\
\hline & 3 & $15.53 \pm 0.05$ & $14.97 \pm 0.04$ & $14.55 \pm 0.04$ \\
\hline & 4 & $16.67 \pm 0.05$ & $15.94 \pm 0.04$ & $15.43 \pm 0.04$ \\
\hline $3 \mathrm{C} 273$ & 1 & $14.14 \pm 0.04$ & $13.53 \pm 0.04$ & $13.14 \pm 0.03$ \\
\hline $3 \mathrm{C} 279$ & 1 & $16.76 \pm 0.04$ & $15.92 \pm 0.03$ & $15.35 \pm 0.03$ \\
\hline \multirow[t]{4}{*}{ PKS 1406-076 } & 1 & $17.67 \pm 0.05$ & $16.89 \pm 0.03$ & $16.31 \pm 0.03$ \\
\hline & 2 & $17.96 \pm 0.05$ & $17.23 \pm 0.04$ & $16.71 \pm 0.03$ \\
\hline & 3 & $16.55 \pm 0.05$ & $15.74 \pm 0.03$ & $15.22 \pm 0.03$ \\
\hline & 4 & $16.04 \pm 0.04$ & $15.40 \pm 0.05$ & $14.96 \pm 0.03$ \\
\hline \multirow[t]{5}{*}{ PKS 1510-089 } & 1 & $13.82 \pm 0.04$ & $13.29 \pm 0.03$ & $12.96 \pm 0.02$ \\
\hline & 2 & $15.16 \pm 0.05$ & $14.44 \pm 0.03$ & $13.98 \pm 0.03$ \\
\hline & 3 & $15.32 \pm 0.04$ & $14.66 \pm 0.03$ & $14.24 \pm 0.03$ \\
\hline & 4 & $15.55 \pm 0.04$ & $14.82 \pm 0.03$ & $14.36 \pm 0.03$ \\
\hline & 5 & $16.18 \pm 0.04$ & $15.23 \pm 0.03$ & $14.59 \pm 0.02$ \\
\hline \multirow[t]{5}{*}{ PKS 1622-297 } & 1 & $17.79 \pm 0.06$ & $16.66 \pm 0.05$ & $16.00 \pm 0.03$ \\
\hline & 2 & $17.83 \pm 0.06$ & $16.68 \pm 0.05$ & $15.89 \pm 0.05$ \\
\hline & 3 & $18.21 \pm 0.06$ & $17.06 \pm 0.05$ & $16.24 \pm 0.04$ \\
\hline & 4 & $18.16 \pm 0.06$ & $17.04 \pm 0.04$ & $16.20 \pm 0.03$ \\
\hline & 5 & $17.41 \pm 0.05$ & $16.59 \pm 0.05$ & $16.04 \pm 0.03$ \\
\hline \multirow[t]{5}{*}{ PKS 1730-130 } & 1 & $16.11 \pm 0.04$ & $14.50 \pm 0.03$ & $13.46 \pm 0.03$ \\
\hline & 2 & $15.71 \pm 0.05$ & $14.28 \pm 0.03$ & $13.37 \pm 0.03$ \\
\hline & 3 & $16.83 \pm 0.05$ & $15.36 \pm 0.03$ & $14.41 \pm 0.03$ \\
\hline & 4 & $15.76 \pm 0.05$ & $14.68 \pm 0.03$ & $13.98 \pm 0.03$ \\
\hline & 5 & $16.60 \pm 0.05$ & $15.05 \pm 0.04$ & $14.04 \pm 0.02$ \\
\hline PKS 2155-304 & 1 & $15.97 \pm 0.03$ & $15.33 \pm 0.04$ & $14.94 \pm 0.03$ \\
\hline \multirow[t]{5}{*}{$3 \mathrm{C} 454.3$} & 1 & $16.76 \pm 0.05$ & $15.87 \pm 0.04$ & $15.29 \pm 0.04$ \\
\hline & 2 & $15.82 \pm 0.05$ & $15.17 \pm 0.05$ & $14.74 \pm 0.03$ \\
\hline & 3 & $16.89 \pm 0.05$ & $15.71 \pm 0.05$ & $14.92 \pm 0.03$ \\
\hline & 4 & $14.57 \pm 0.05$ & $13.65 \pm 0.04$ & $13.09 \pm 0.03$ \\
\hline & 5 & $16.65 \pm 0.05$ & $15.77 \pm 0.04$ & $15.20 \pm 0.04$ \\
\hline
\end{tabular}

Note. Errors are $1 \sigma$.

(see Figure 7). Field of view is approximately $6^{\prime} \times 6^{\prime}$. North is at top of image, east is to the left. Comparison stars are numbered; calibrated magnitudes for our comparison stars are given in Appendix C.

\section{APPENDIX B}

\section{NEAR-INFRARED FINDING CHARTS}

We present near-infrared finding charts for each of the 12 SMARTS-monitored blazars discussed in this paper. All figures are $J$-band (Figure 8). Field of view is approximately $2^{\prime} \times 2^{\prime}$. North is to the right of image, east is at the top. Comparison stars are numbered; calibrated magnitudes for our comparison stars are given in Appendix C. 
Table 4

Comparison Star Near-infrared Magnitudes

\begin{tabular}{|c|c|c|c|}
\hline Source & Star & $J$ & $K$ \\
\hline PKS 0208-512 & 1 & $12.16 \pm 0.04$ & $11.81 \pm 0.07$ \\
\hline \multirow[t]{2}{*}{ AO $0235+164$} & 1 & $11.96 \pm 0.04$ & $11.68 \pm 0.06$ \\
\hline & 2 & $12.97 \pm 0.04$ & $12.14 \pm 0.09$ \\
\hline \multirow[t]{3}{*}{ PKS 0528+134 } & 1 & $14.03 \pm 0.08$ & \\
\hline & 2 & $12.72 \pm 0.06$ & \\
\hline & 3 & $12.32 \pm 0.05$ & \\
\hline OJ 287 & 1 & $14.58 \pm 0.080$ & $13.79 \pm 0.10$ \\
\hline \multirow[t]{2}{*}{$3 C 273$} & 1 & $13.64 \pm 0.06$ & \\
\hline & 2 & $12.29 \pm 0.05$ & \\
\hline \multirow[t]{2}{*}{$3 C 279$} & 1 & $14.41 \pm 0.07$ & $13.86 \pm 0.12$ \\
\hline & 2 & $15.47 \pm 0.14$ & $13.94 \pm 0.20$ \\
\hline \multirow[t]{2}{*}{ PKS 1406-076 } & 1 & $15.32 \pm 0.07$ & $14.84 \pm 0.16$ \\
\hline & 2 & $15.77 \pm 0.11$ & $15.30 \pm 0.47$ \\
\hline \multirow[t]{3}{*}{ PKS 1510-089 } & 1 & $15.61 \pm 0.13$ & $14.71 \pm 0.27$ \\
\hline & 2 & $15.57 \pm 0.11$ & $14.44 \pm 0.32$ \\
\hline & 3 & $13.49 \pm 0.05$ & $12.77 \pm 0.12$ \\
\hline \multirow[t]{3}{*}{ PKS 1622-297 } & 1 & $14.43 \pm 0.11$ & $13.47 \pm 0.14$ \\
\hline & 2 & $13.83 \pm 0.11$ & $12.69 \pm 0.12$ \\
\hline & 3 & $13.53 \pm 0.08$ & $12.67 \pm 0.11$ \\
\hline \multirow[t]{3}{*}{ PKS 1730-130 } & 1 & $14.37 \pm 0.07$ & $13.17 \pm 0.10$ \\
\hline & 2 & $13.06 \pm 0.07$ & $11.96 \pm 0.06$ \\
\hline & 3 & $14.60 \pm 0.07$ & $13.48 \pm 0.10$ \\
\hline PKS 2155-304 & 1 & $14.30 \pm 0.06$ & $13.72 \pm 0.14$ \\
\hline \multirow[t]{2}{*}{$3 \mathrm{C} 454.3$} & 1 & $14.26 \pm 0.10$ & $13.49 \pm 0.15$ \\
\hline & 2 & $11.88 \pm 0.07$ & $11.31 \pm 0.61$ \\
\hline
\end{tabular}

Note. Errors are $1 \sigma$.

\section{APPENDIX C}

\section{COMPARISON STAR MAGNITUDES}

Here we present calibrated magnitudes for comparison stars indicated in the finding charts presented in Appendices A and B used as secondary standards for our differential photometry.

The error in calibrating the secondary star magnitudes was found by calculating the $1 \sigma$ standard error of the mean over the number of photometric nights on which data for the star were taken. Results that were greater than $\pm 3 \sigma$ from the mean were rejected and the mean and $\sigma$ were recalculated. This procedure was repeated until no more rejections were made. The resulting $1 \sigma$ errors are given in the following tables. Table 3 gives $B V R$ magnitudes for comparison stars used in optical photometry. Table 4 gives $J K$ magnitudes for comparison stars used in the infrared photometry.

\section{REFERENCES}

Abdo, A. A., Ackermann, M., Ajello, M., et al. 2010, Nature, 463, 919

Agudo, I., Marscher, A. P., Jorstad, S. G., et al. 2011, ApJ, 735, L10

Alcock, C., Allsman, R. A., Alves, D. R., et al. 2000, ApJ, 542, 281

Angione, R. J. 1971, AJ, 76, 412

Beckwith, S., Evans, N. J., II, Becklin, E. E., \& Neugebauer, G. 1976, ApJ, 208 , 390

Bessell, M. S., Castelli, F., \& Plez, B. 1998, A\&A, 333, 231

Bonning, E. W., Bailyn, C., Urry, C. M., et al. 2009, ApJ, 697, L81

Bonnoli, G., Ghisellini, G., Foschini, L., Tavecchio, F., \& Ghirlanda, G. 2011, MNRAS, 410, 368

Böttcher, M. 2007, Ap\&SS, 309, 95

Burbidge, E. M., Beaver, E. A., Cohen, R. D., Junkkarinen, V. T., \& Lyons, R. W. 1996, AJ, 112, 2533
Buxton, N. M., Bailyn, C. D., Capelo, H. L., et al. 2012, AJ, 143, 130

Cardelli, J. A., Clayton, G. C., \& Mathis, J. S. 1989, ApJ, 345, 245

Cohen, R. D., Smith, H. E., Junkkarinen, V. T., \& Burbidge, E. M. 1987, ApJ, 318, 577

Coppi, P. S. 1992, MNRAS, 258, 657

Dai, B. Z., Li, X. H., Liu, Z. M., et al. 2009, MNRAS, 392, 1181

DePoy, D. L., Atwood, B., Belville, S. R., et al. 2003, Proc. SPIE, 4841, 827

Dermer, C. D., \& Schlickeiser, R. 1993, ApJ, 416, 458

Dolcini, A., Farfanelli, F., Ciprini, S., et al. 2007, A\&A, 469, 503

Edelson, R., Turner, T. J., Pounds, K., et al. 2002, ApJ, 568, 610

Edelson, R. A., \& Krolik, J. H. 1988, ApJ, 333, 646

Fiorucci, M., \& Tosti, G. 1996, A\&AS, 116, 403

Fiorucci, M., Tosti, G., \& Rizzi, N. 1998, PASP, 110, 105

Fossati, G., Maraschi, L., Celotti, A., Comastri, A., \& Ghisellini, G. 1998, MNRAS, 299, 433

Ghisellini, G., \& Celotti, A. 2001, MNRAS, 327, 739

Ghisellini, G., Foschini, L., Tavecchio, F., \& Pian, E. 2007, MNRAS, 382, L82

Ghisellini, G., \& Madau, P. 1996, MNRAS, 280, 67

Ghisellini, G., \& Tavecchio, F. 2010, MNRAS, 409, L79

González-Pérez, J. N., Kidger, M. R., \& Martín-Luis, F. 2001, AJ, 122, 2055

Gu, M. F., Lee, C.-U., Pak, S., Yim, H. S., \& Fletcher, A. B. 2006, A\&A, 450, 39

Hagen-Thorn, V. A., Marchenko, S. G., Takalo, L. O., et al. 1998, A\&AS, 133, 353

Hamuy, M., \& Maza, J. 1989, AJ, 97, 720

Hartman, R. C., Webb, J. R., Marscher, A. P., et al. 1996, ApJ, 461, 698

Jones, T. W., O'dell, S. L., \& Stein, W. A. 1974, ApJ, 188, 353

Junkkarinen, V. T., Cohen, R. D., Beaver, E. A., et al. 2004, ApJ, 614, 658

Kataoka, J., Takahashi, T., Makino, F., et al. 2000, ApJ, 528, 243

Konigl, A. 1981, ApJ, 243, 700

Landolt, A. U. 1992, AJ, 104, 340

Lehto, H. J., \& Valtonen, M. J. 1996, ApJ, 460, 207

Maccarone, T. J., \& Coppi, P. S. 2003, MNRAS, 338, 189

Malkan, M. A. 1983, ApJ, 268, 582

Marscher, A. P., Jorstad, S. G., Larionov, V. M., et al. 2010, ApJ, 710, L126

Moderski, R., Sikora, M., Coppi, P. S., \& Aharonian, F. 2005a, MNRAS, 364, 1488

Moderski, R., Sikora, M., Coppi, P. S., \& Aharonian, F. 2005b, MNRAS, 363, 954

Monet, D. G., Levine, S. E., Canzian, B., et al. 2003, AJ, 125, 984

Mücke, A., \& Protheroe, R. J. 2001, Astropart. Phys., 15, 121

Mücke, A., Protheroe, R. J., Engel, R., Rachen, J. P., \& Stanev, T. 2003 , Astropart. Phys., 18, 593

Nandra, K., George, I. M., Mushotzky, R. F., Turner, T. J., \& Yaqoob, T. 1997, ApJ, 476, 70

Nilsson, K., Charles, P. A., Pursimo, T., et al. 1996, A\&A, 314, 754

Osterman Meyer, A., Miller, H. R., Marshall, K., et al. 2008, AJ, 136, 1398

Paltani, S., Courvoisier, T. J.-L., Blecha, A., \& Bratschi, P. 1997, A\&A, 327, 539

Persson, S. E., Murphy, D. C., Krzeminski, W., Roth, M., \& Rieke, M. J. 1998, AJ, 116, 2475

Pian, E., Edelson, R. A., Wagner, S. J., et al. 1995, Adv. Space Res., 16, 57

Pian, E., Vacanti, G., Tagliaferri, G., et al. 1998, ApJ, 492, L17

Raiteri, C. M., Villata, M., Ibrahimov, M. A., et al. 2005, A\&A, 438, 39

Raiteri, C. M., Villata, M., Kadler, M., et al. 2006, A\&A, 459, 731

Raiteri, C. M., Villata, M., Lanteri, L., Cavallone, M., \& Sobrito, G. 1998 A\&AS, 130, 495

Raiteri, C. M., Villata, M., Larionov, V. M., et al. 2007, A\&A, 473, 819

Raiteri, C. M., Villata, M., Larionov, V. M., et al. 2008, A\&A, 480, 339

Ramos, E., Kafatos, M., Fruscione, A., et al. 1997, ApJ, 482, 167

Rani, B., Gupta, A. C., Strigachev, A., et al. 2010, MNRAS, 404, 1992

Remillard, R. A., \& McClintock, J. E. 2006, ARA\&A, 44, 49

Schlegel, D. J., Finkbeiner, D. P., \& Davis, M. 1998, ApJ, 500, 525

Sikora, M., Begelman, M. C., \& Rees, M. J. 1994, ApJ, 421, 153

Sikora, M., \& Madejski, G. 2000, ApJ, 534, 109

Sillanpaa, A., Haarala, S., Valtonen, M. J., Sundelius, B., \& Byrd, G. G. 1988, ApJ, 325, 628

Sillanpaa, A., Takalo, L. O., Pursimo, T., et al. 1996, A\&A, 315, L13

Smith, D. M., Dawson, D. M., \& Swank, J. H. 2007, ApJ, 669, 1138

Smith, P. S., \& Balonek, T. J. 1998, PASP, 110, 1164

Smith, P. S., Balonek, T. J., Heckert, P. A., Elston, R., \& Schmidt, G. D. 1985, AJ, 90,1184

Sun, W., \& Malkan, M. A. 1989, ApJ, 346, 68

Tavecchio, F., Maraschi, L., Sambruna, R. M., \& Urry, C. M. 2000, ApJ, 544, L23

Urry, C. M., \& Mushotzky, R. F. 1982, ApJ, 253, 38 
Urry, C. M., \& Padovani, P. 1995, PASP, 107, 803

Vaughan, S., Edelson, R., Warwick, R. S., \& Uttley, P. 2003, MNRAS, 345, 1271

Villforth, C., Nilsson, K., Heidt, J., et al. 2010, MNRAS, 402, 2087

Wehrle, A. E., Pian, E., Urry, C. M., et al. 1998, ApJ, 497, 178
White, R. J., \& Peterson, B. M. 1994, PASP, 106, 879

Yanny, B., York, D. G., \& Gallagher, J. S. 1989, ApJ, 338, 735

Zhai, M., Zheng, W. K., \& Wei, J. Y. 2011, A\&A, 531, A90

Zheng, Y. G., Zhang, X., Bi, X. W., Hao, J. M., \& Zhang, H. J. 2008, MNRAS, 385,823 